DEPARTMENT OF COMMERCE

BUREAU OF STANDARDS

George K. Burgess, Director

TECHNOLOGIC PAPERS OF THE BUREAU OF STANDARDS, No. 258

[Part of Vol. 18]

\title{
STRENGTH OF STEEL TUBING UNDER COMBINED COLUMN AND TRANSVERSE \\ LOADING, INCLUDING TESTS OF COLUMNS AND BEAMS
}

BY

TOM W. GREENE, Assistant Engineer Physicist

Bureau of Slandards

May 23, 1924

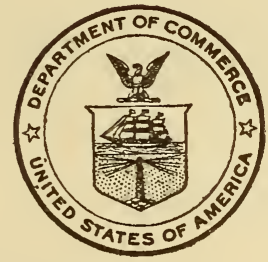

PRICE, 15 CENTS

\$1.25 PER VOLUME ON SUBSCRIPTION

Sold only by the Superintendent of Documents, Government Printing Office Washington, D. C.

WASHINGTON

GOVERNMENT PRINTING OFFICE 



\title{
STRENGTH OF STEEL TUBING UNDER COMBINED COLUMN AND TRANSVERSE LOADING, INCLUDING TESTS OF COLUMNS AND BEAMS.
}

\author{
By Tom W. Greene.
}

\begin{abstract}
.
This investigation was made for the purpose of determining whether experimental data confirmed the theory of struts subjected to combined column and transverse loading. A number of tests were made on steel-tubing struts ranging from that of a column with no transverse load to that of a beam with no column load.

A study was made of the conditions contributing to the strength of a strut and a method devised for measuring eccentricity. It was found that the eccentricity due to variation in wall thickness and to deviation from straightness is an important factor and should be taken into account. The results show that the commonly used formulas, which neglect the effect of eccentricity of loading, do not represent actual strut condition and are liable to give dangerously high results.

A modified rational formula based upon consideration of the effect of eccentricity was found to fit experimental results very closely and is the preferable one for design. Failure of a strut will occur when the maximum compressive stress computed by this modified formula is approximately equal to the yield point. The modified rational formula also applies to columns as it reduces to the "secant" column formula when the transverse load is equated to zero. Failure of a column will occur when the extreme fiber stress computed by the "secant" formula is equal to the yield point of the material.

A reasonably accurate computation of the stress for a strut under transverse load can be made by summing the bending stress due to the transverse load and the column stress obtained by the "secant" formula if for the latter the effective eccentricity is taken as the sum of the original eccentricity, due to tube irregularities, and the deflection of the strut at the center resulting from the transverse load.
\end{abstract}

\section{CONTENTS.}

I. Introduction........................................ 244

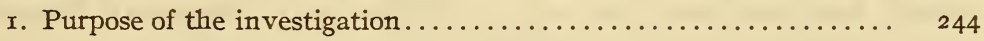

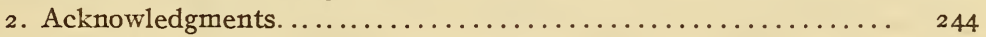

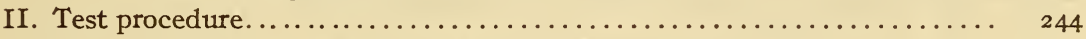

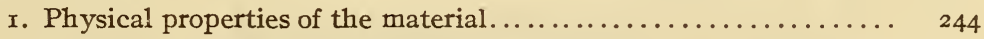

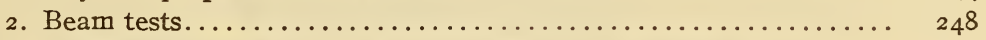

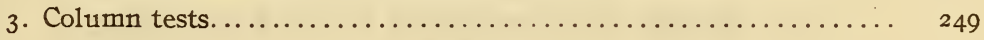

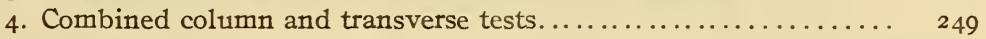

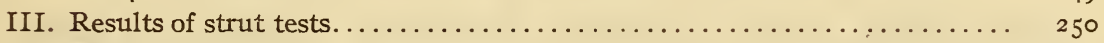

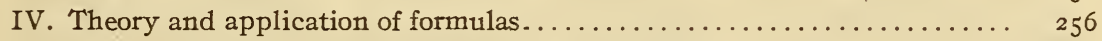

I. Application of formulas for combined loading............. ${ }_{2} 5^{6}$

2. Modified rational formula for combined loading........... 260

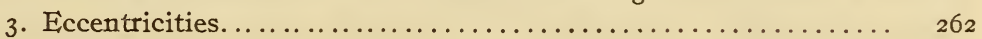


IV. Theory and application of formulas-Continued.

3. Eccentricities-Continued.

(a) Causes of eccentricity......................... 262

(b) Determination of eccentricity................... 262

I. Eccentricity due to variation in wall thickness.... 262

2. Eccentricity due to deviation from straightness... 264

(c) Discussion............................... ${ }_{26} 66_{5}$

(d) Accuracy of method of determining eccentricity......... 268

4. Application of modified rational formula ................. 269

5. Application of "secant" column formula................ 27 I

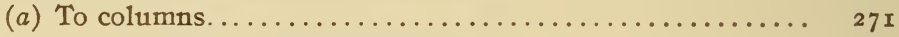

(b) To struts with transverse loading............... 272

V. Conclusions................................... ${ }^{275}$

VI. Recommendations.................................... ${ }_{27} 6$

\section{INTRODUCTION.}

\section{PURPOSE OF THE INVESTIGATION.}

In airplane construction there are many members, such as struts, which are subjected to a lateral or transverse loading in addition to an axial or column loading. This investigation was made at the request of the Bureau of Aeronautics, Navy Department, for the purpose of determining whether experimental data confirmed the approximate theory of struts subjected to combined axial and transverse forces or whether it would be necessary to devise new formulas.

A large number of tests were made on steel-tubing struts of different lengths with various intensities of transverse loading. The tests included different ratios of direct compression to transverse loading, ranging from that of a column with no transverse load to that of a beam with no column load.

\section{ACKNOWLEDGMENTS.}

The funds and material for this investigation were furnished by the Bureau of Aeronautics, Navy Department. Acknowledgments are also due Lieut. C. J. McCarthy, Bureau of Aeronautics, for his assistance, cooperation, and suggestions.

\section{TEST PROCEDURE.}

\section{PHYSICAL PROPERTIES OF THE MATERIAL.}

Steel tubing made in England of $\mathrm{I} / 2$-inch diameter, 20 gauge, and $1 / 2$-inch diameter, 16 gauge, was used for all struts and beams in this investigation. The physical properties of the material were accurately determined by tensile and compression tests of 
short specimens cut from every 15 -foot length or section of tubing. The physical properties of each section of tubing used are given in Table I, and a few typical stress strain curves are shown in Figure I.

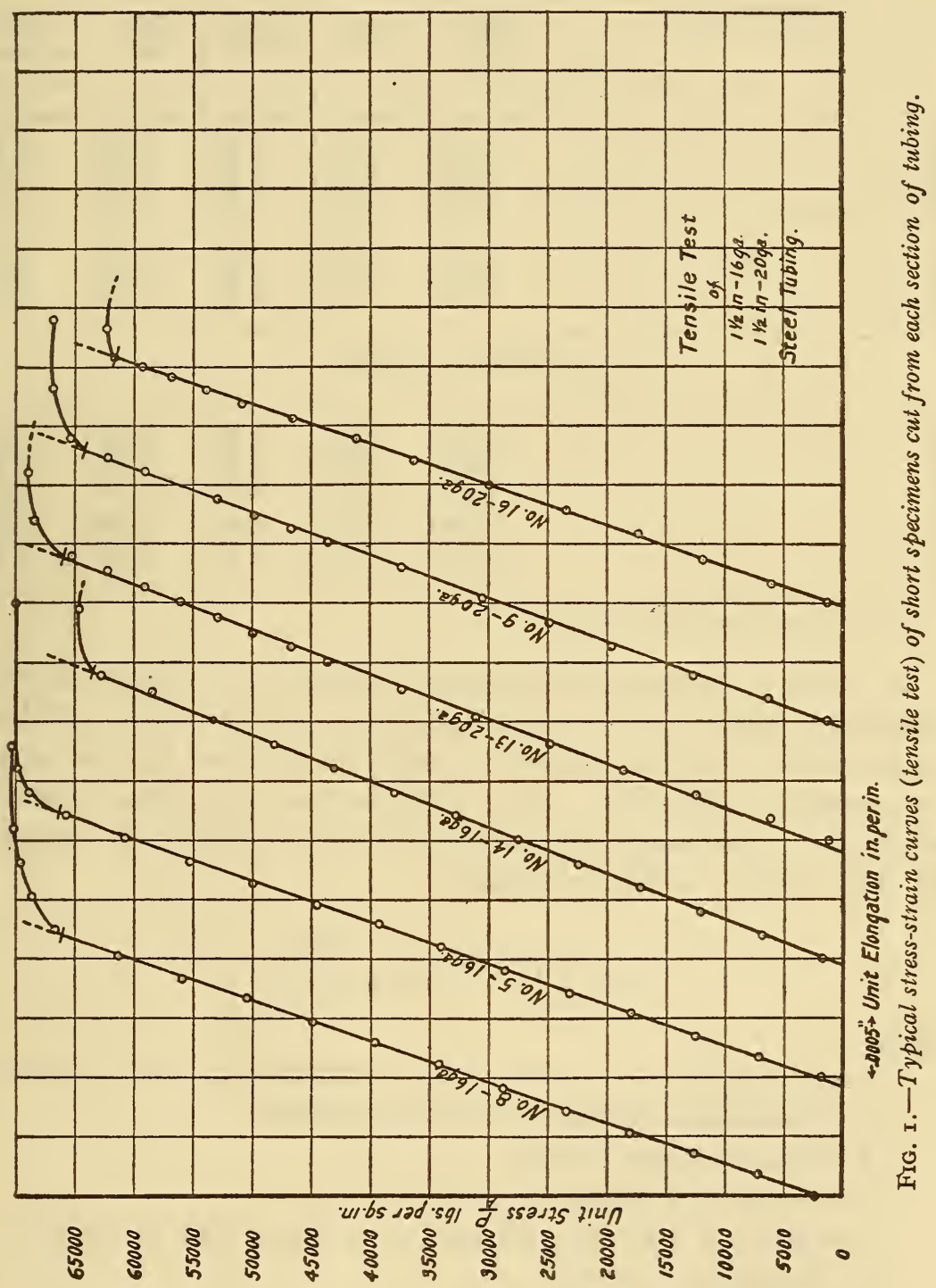

A comparison of the yield point values in tension with the ultimate compressive stress at crinkling shows considerable variations. It is believed that a more accurate determination of the-ultimate compressive stress to cause failure for a short column is given by the "secant" column formula in which the effect of eccentricity of loading is considered. 
TABLE 1.-Physical Properties of Each Section of Tubing.

$1 \% / 2$ INCHES, 20 GAUGE.

\begin{tabular}{|c|c|c|c|c|c|}
\hline \multirow[b]{2}{*}{ Section number. } & \multicolumn{3}{|c|}{ Tension. } & \multicolumn{2}{|c|}{ Compression. ${ }^{1}$} \\
\hline & $\begin{array}{l}\text { Propor- } \\
\text { tional } \\
\text { limit. }\end{array}$ & $\begin{array}{l}\text { Yield } \\
\text { point. }\end{array}$ & $\begin{array}{l}\text { Ultimate } \\
\text { strength. }\end{array}$ & $\begin{array}{c}\text { Propor- } \\
\text { tional } \\
\text { limit. }\end{array}$ & $\begin{array}{c}\text { Ultimate } \\
\text { stress } \\
\text { (crinkling). }\end{array}$ \\
\hline 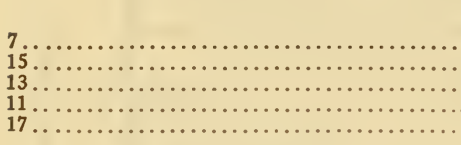 & $\begin{array}{c}\text { Lbs./in. }{ }^{2} \\
66,000 \\
64,000 \\
66,000 \\
67,500 \\
66,000\end{array}$ & $\begin{array}{r}\text { Lbs./in. }{ }^{2} \\
72,100 \\
69,900 \\
68,900 \\
69,000 \\
67,400\end{array}$ & $\begin{array}{r}\text { Lbs./in. }{ }^{2} \\
78,900 \\
76,400 \\
74,900 \\
74,000 \\
73,400\end{array}$ & $\begin{array}{r}\text { Lbs./in.2 } \\
58,500 \\
54,000 \\
56,500 \\
58,000 \\
56,000\end{array}$ & $\begin{array}{c}\text { Lbs./in. }{ }^{2} \\
67,700 \\
65,800 \\
65,000 \\
64,800 \\
68,400\end{array}$ \\
\hline $\begin{array}{l}9 \\
4 \ldots \ldots \ldots \ldots \ldots \\
1 \\
16 \ldots \ldots \ldots \cdots \cdots\end{array}$ & $\begin{array}{l}64,000 \\
54,000 \\
59,000 \\
61,500\end{array}$ & $\begin{array}{l}66,800 \\
63,400 \\
61,200 \\
62,200\end{array}$ & $\begin{array}{l}73,400 \\
70,800 \\
69,600 \\
68,700\end{array}$ & $\begin{array}{l}56,000 \\
53,000 \\
53,500 \\
52,600\end{array}$ & $\begin{array}{l}64,800 \\
65,200 \\
59,000 \\
66,200\end{array}$ \\
\hline
\end{tabular}

$11 / 2$ INCHES, 16 GAUGE.

\begin{tabular}{|c|c|c|c|c|c|}
\hline 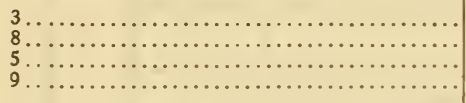 & $\begin{array}{l}67,000 \\
66,000 \\
66,000 \\
65,000\end{array}$ & $\begin{array}{l}71,300 \\
70,200 \\
70,000 \\
69,100\end{array}$ & $\begin{array}{l}75,300 \\
74,600 \\
73,800 \\
73,400\end{array}$ & $\begin{array}{l}59,000 \\
54,000 \\
55,000 \\
55,000\end{array}$ & $\begin{array}{l}75,500 \\
71,800 \\
67,800 \\
69,500\end{array}$ \\
\hline 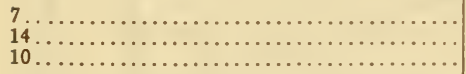 & $\begin{array}{l}62,000 \\
63,500 \\
59,000\end{array}$ & $\begin{array}{l}67,500 \\
64,600 \\
61,300\end{array}$ & $\begin{array}{l}71,400 \\
69,600 \\
67,100\end{array}$ & $\begin{array}{l}54,000 \\
51,000 \\
51,000\end{array}$ & $\begin{array}{l}67,400 \\
65,300 \\
65,800\end{array}$ \\
\hline
\end{tabular}

${ }^{1} L / r=$ ro for short compression specimens.

In Table 2 are given the eccentricities due to variation in wall thickness, which will be explained later, and the effect of these eccentricities on the ultimate compressive stress for the short compression specimens. The values in the last column are the ultimate or maximum compressive stresses at failure $S_{\mathrm{c}}$ obtained by the "secant" column formula,

where

$$
S_{\mathrm{c}}=\frac{P}{A}\left(\mathrm{I}+\frac{e c}{r^{2}} \sec \sqrt{\frac{P L^{2}}{4 E I}}\right)
$$

$e=$ eccentricity in inches due to variation in wall thickness,

$P=$ maximum compressive load in pounds,

$A=$ area in square inches,

$r=$ radius of gyration in inches,

$c=$ distance from neutral axis to extreme fiber in inches,

$L=$ length in inches,

$I=$ moment of inertia,

$E=$ modulus of elasticity $\left(29,000,000 \mathrm{lbs} . /\right.$ in. $\left.{ }^{2}\right)$. 



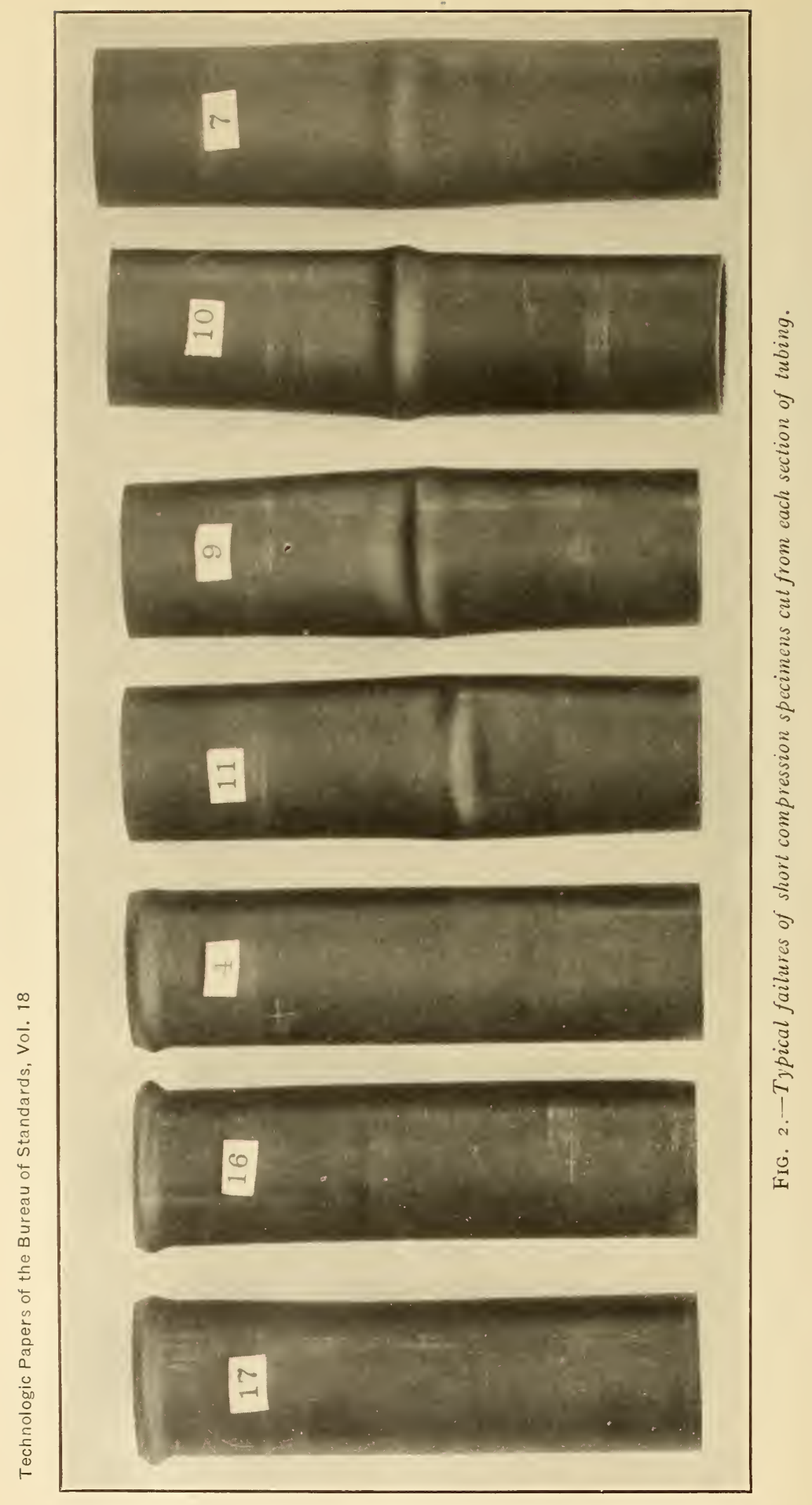


TABLE 2.-Ultimate or Maximum Compressive Stresses for Short Compression Specimens.

11/2 INCHES, 20 GAUGE.

\begin{tabular}{|c|c|c|c|}
\hline Section number. & $\begin{array}{l}\text { Variation in } \\
\text { thickness of } \\
\text { wall. }\end{array}$ & $\begin{array}{c}\text { Eccen- } \\
\text { tricity. } \\
e\end{array}$ & $\begin{array}{l}\text { Ultimate } \\
\text { compres- } \\
\text { sive stress } \\
\text { at failure. } \\
S_{\mathrm{c}} 1\end{array}$ \\
\hline 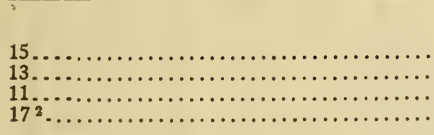 & $\begin{array}{r}\text { Inches. } \\
0.0325-0.035 \\
.033-.037 \\
.0325-.035 \\
.035\end{array}$ & $\begin{array}{c}\text { Inches. } \\
0.0153 \\
.022 \\
.014 \\
.000\end{array}$ & $\begin{array}{r}\text { Lbs./in.2 } \\
68,800 \\
69,300 \\
67,600 \\
68,400\end{array}$ \\
\hline 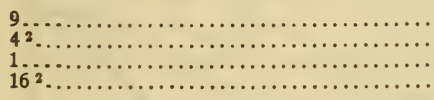 & $\begin{array}{r}.0345-.0355 \\
.035 \\
.034-.040 \\
.036\end{array}$ & $\begin{array}{l}.0083 \\
.000 \\
.032 \\
.000\end{array}$ & $\begin{array}{l}66,400 \\
65,200 \\
64,700 \\
66,200\end{array}$ \\
\hline
\end{tabular}

11/2 INCHES, 16 GAUGE.

\begin{tabular}{|c|c|c|c|}
\hline 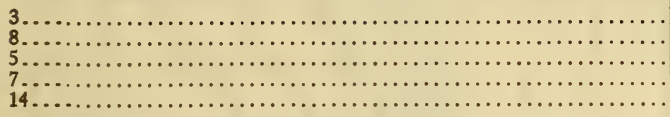 & $\begin{array}{l}0.059-0.066 \\
.0595-.064 \\
.059-.066 \\
.060-.063 \\
.061-.067\end{array}$ & $\begin{array}{l}0.0215 \\
.014 \\
.0215 \\
.0095 \\
.018\end{array}$ & $\begin{array}{l}80,400 \\
74,800 \\
71,600 \\
69,300 \\
68,800\end{array}$ \\
\hline
\end{tabular}

${ }^{1} S_{0}=\frac{P}{A}\left(\mathrm{I}+\frac{e c}{t^{2}} \sec \sqrt{\frac{P L^{2}}{4 E I}}\right)$ where $e$ is eccentricity due to variation in wall thickness and $P$ is the maximum compressive load (crinkling).

2 Short compressive specimens crinkled near end.

It will be seen that for tubes with eccentricity of loading the values for the ultimate stress in compression are raised and approximate very closely the yield point of the material in tension. Although the "secant" column formula is not exact above the proportional limit, it is believed that for the material used, in which the proportional limit and yield point are nearly the same, the ultimate compressive stress value $S_{\mathrm{c}}$ in the table is probably the yield point in compression and represents very closely the stress to cause failure in compression for any strut cut from these sections.

The effect of eccentricity is also indicated in the type of failure for the short columns. In specimens Nos. I7, 4, and I6, where the eccentricities were zero, the specimens failed by crinkling near the ends. In the other short columns the crinkling occurred on the thin side at the center where the maximum compressive stress occurs from bending due to the eccentricities. Figure 2 shows a few of the typical failures. 


\section{BEAM TESTS.}

To obtain conditions where the bending stress is a maximum with no column load, transverse tests were made on three specimens of each gauge thickness. The tubing was tested as a simple

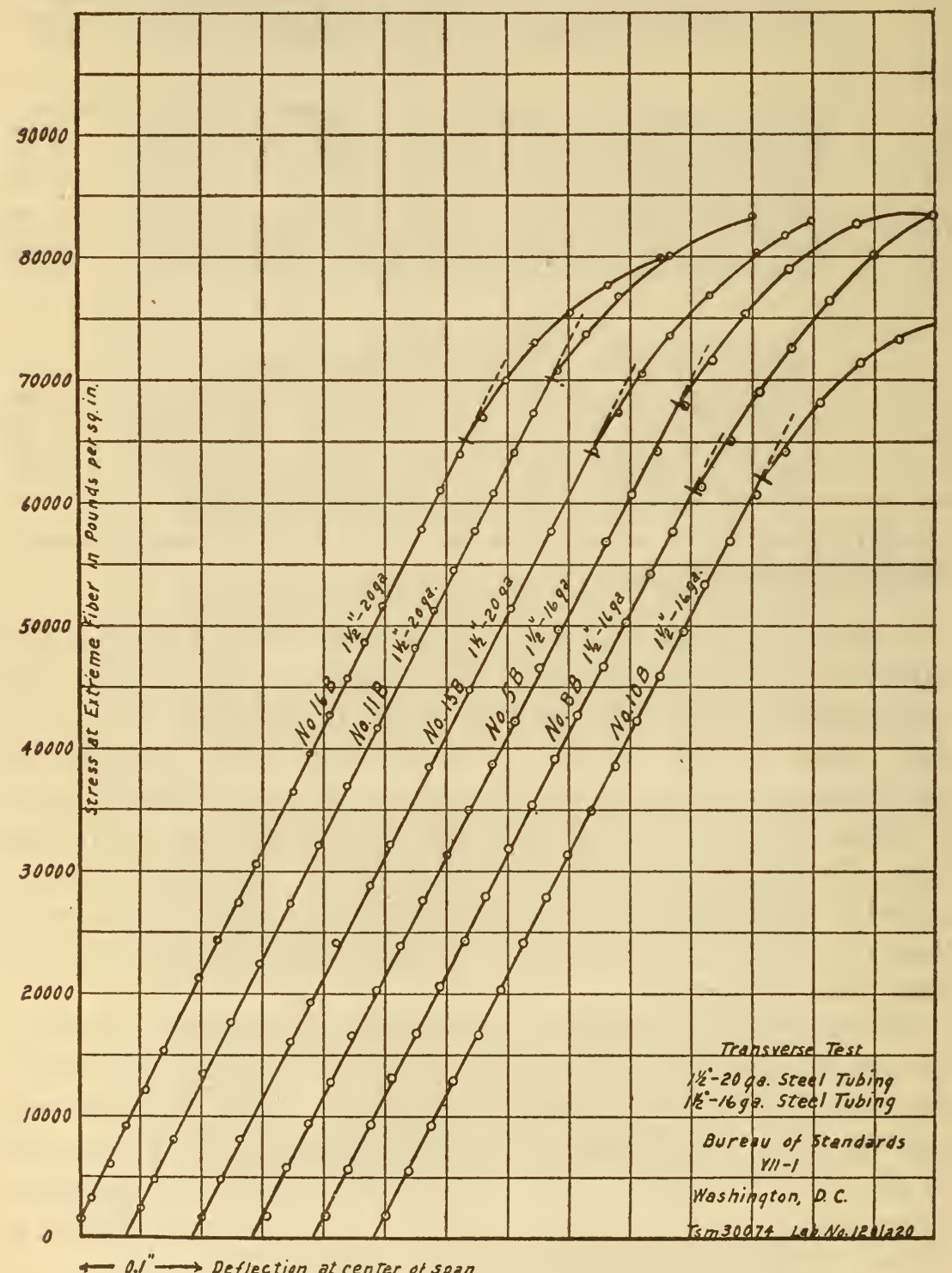

FIG. 3.-Stress-deflection curves, transverse test.

beam of 36-inch span. The load was applied at the center of the span by means of a wooden block one-half inch thick cut to fit the tubing. The results of the beam tests are given in Table 3 , and the stress deflection curves are shown plotted in Figure 3. 


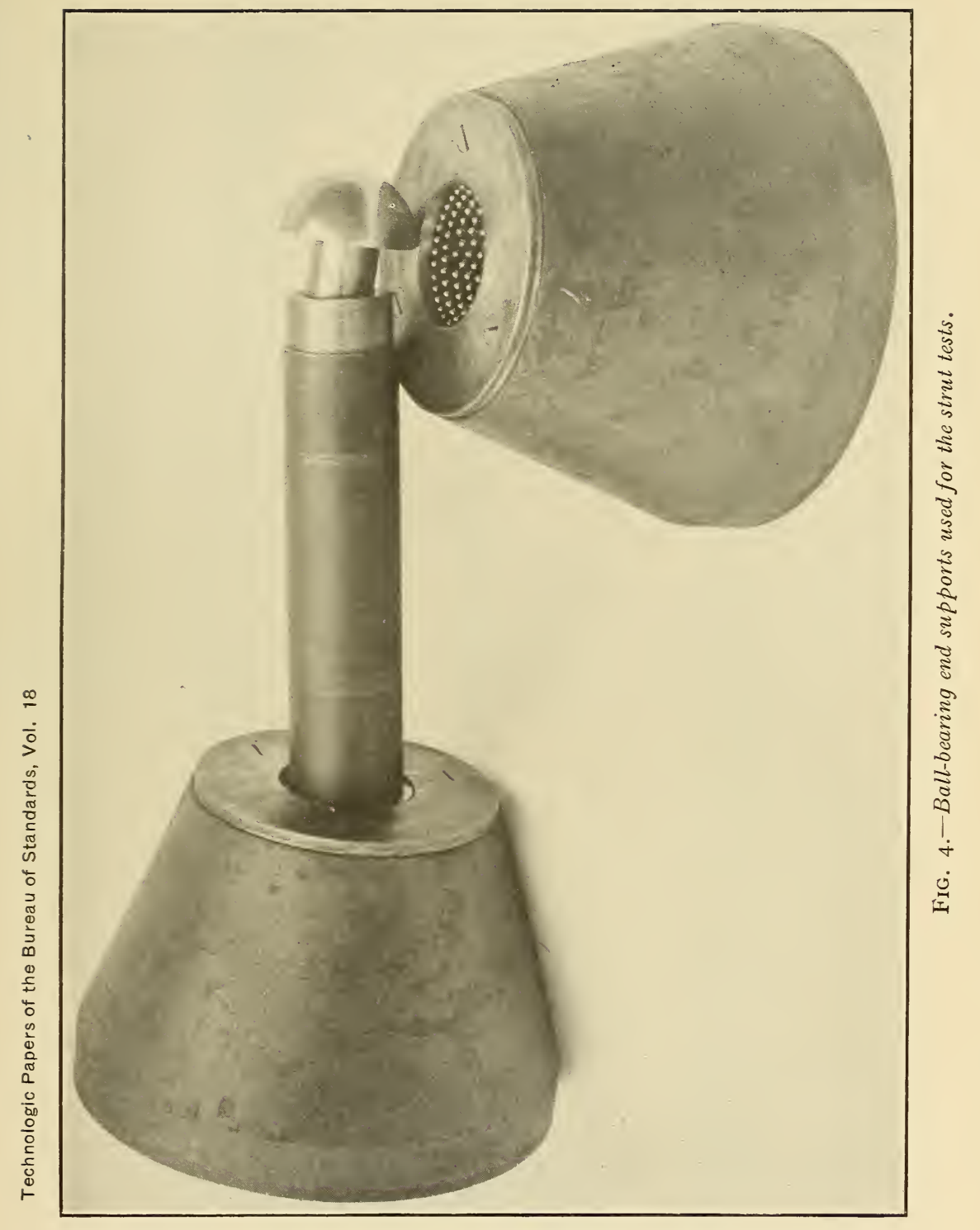




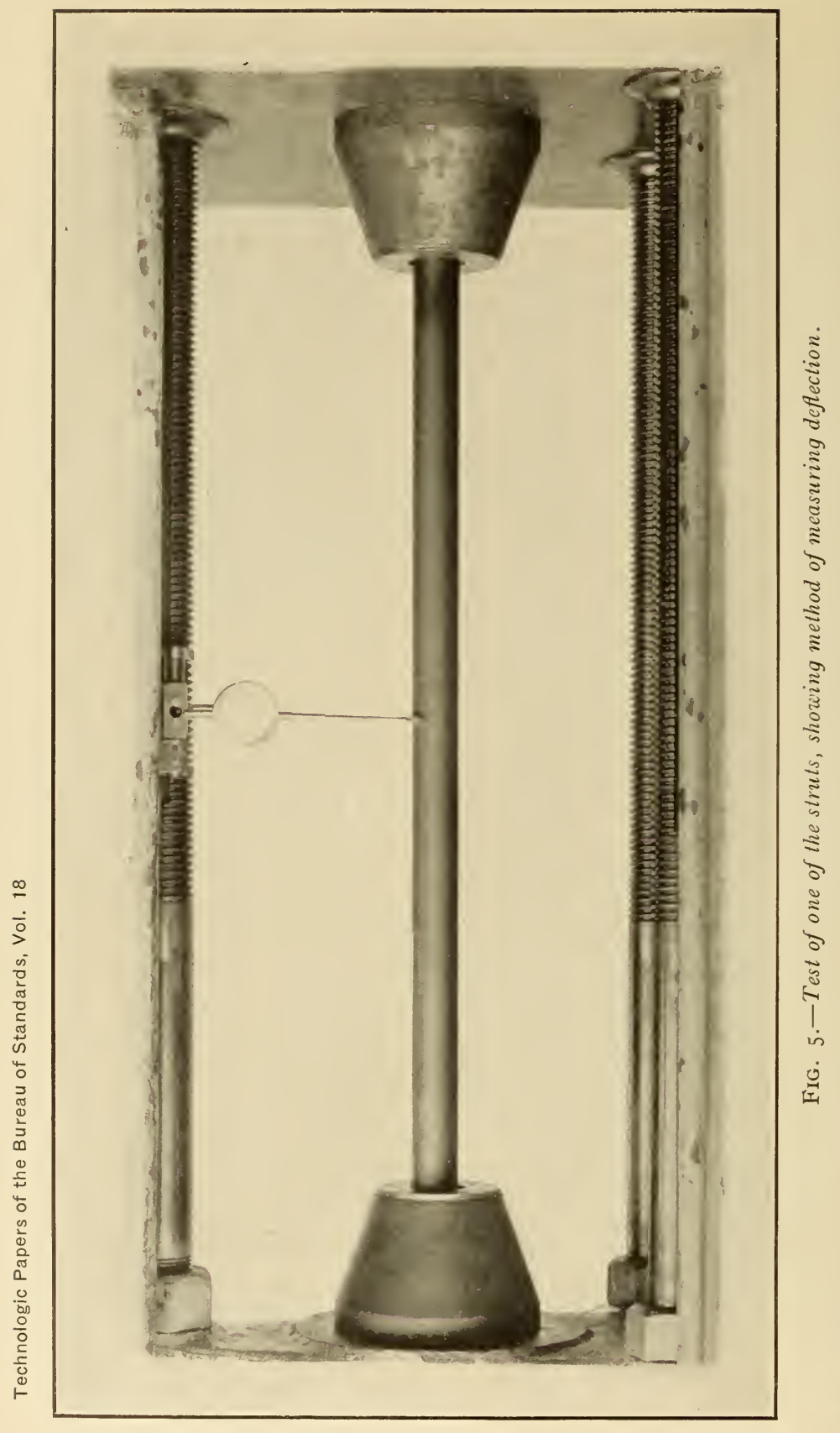


TABLE 3.-Beam Test (Span 36 Inches).

$11 / 2$ INCHES, 20 GAUGE.

\begin{tabular}{|c|c|c|c|c|c|c|c|}
\hline $\begin{array}{l}\text { Specimen } \\
\text { number. }\end{array}$ & $\begin{array}{c}\text { Variation in } \\
\text { thickness of } \\
\text { wall. }\end{array}$ & Area. & $\begin{array}{l}\text { Moment } \\
\text { of inertia. } \\
I\end{array}$ & $\begin{array}{l}\text { Sectional } \\
\text { modulus. } \\
\qquad \frac{I}{c}\end{array}$ & $\begin{array}{l}\text { Propor- } \\
\text { tional } \\
\text { limit. }\end{array}$ & $\begin{array}{l}\text { Modulus } \\
\text { of rupture. }\end{array}$ & $\begin{array}{l}\text { Modulus of } \\
\text { elasticity. }\end{array}$ \\
\hline 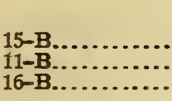 & $\begin{array}{c}\text { Inches. } \\
0.032-0.035 \\
.033-.035 \\
.035-.037\end{array}$ & $\begin{array}{r}\text { Sq. inch. } \\
0.1566 \\
.1566 \\
.1656\end{array}$ & $\begin{array}{l}\text { In.4 } \\
0.0421 \\
.0421 \\
.0444\end{array}$ & $\begin{array}{l}\text { In. }{ }^{3} \\
0.0562 \\
.0562 \\
.0592\end{array}$ & $\begin{array}{r}\text { Lbs./in. }^{2} \\
64,000 \\
70,000 \\
65,000\end{array}$ & $\begin{array}{r}\text { Lbs./in. }{ }^{2} \\
88,080 \\
85,670 \\
82,080\end{array}$ & $\begin{array}{l}\text { Lbs./in.2 } \\
28,400,000 \\
29,020,000 \\
29,140,000\end{array}$ \\
\hline
\end{tabular}

11/2 INCHES, 16 GAUGE.

\begin{tabular}{r|r|r|r|r|r|r|r}
\hline $8-\mathrm{B} \ldots \ldots \ldots \ldots .$. & $0.061-0.063$ & 0.2803 & 0.0725 & 0.0966 & 61,000 & 92,600 & $28,080,000$ \\
$5-\mathrm{B} \ldots \ldots \ldots \ldots .$. & $.061-.065$ & .2846 & .0735 & .0980 & 68,000 & 91,800 & $27,500,000$ \\
$10-\mathrm{B} \ldots \ldots \ldots \ldots .$. & $.062-.064$ & .2846 & .0735 & .0980 & 62,000 & 83,560 & $28,080,000$ \\
\hline
\end{tabular}

\section{COLUMN TESTS.}

Some of the tubes were tested as round end columns without transverse loading. Free end conditions were insured by special ball-bearing end supports. Figure 4 shows the ball-bearing end supports used for all the compression tests. The small balls minimized the frictional resistance developed during the loading of the struts and permitted the struts to deflect freely in any direction, thus approaching ideal "free end" conditions.

\section{COMBINED COLUMN AND TRANSVERSE TESTS.}

The tube was mounted as in the column test for the combined beam and column tests. These tests were made in a horizontal position in an Emery hydraulic testing machine. Extreme care and precaution was exercised in the application of the side load. A small initial end load was first applied to the strut. The transverse load was then applied. For uniform transverse loads of I, 5 , IO, and 20 pounds per linear inch weights of I, 5 , IO, and 20 pounds, respectively, were suspended I inch apart throughout the length of the struts. For loads of I.25, 3, and 6.05 pounds per linear inch weights were suspended in bags attached to the tubing. Thus, a very uniform distribution of lateral loading was obtained in all the tests.

Measurements of the original straightness of the tubes and of the vertical deflection under load at the mid length of the test piece were taken for all the tests by means of a micrometer dial reading directly to $0.00 \mathrm{I}$ inch. Figure 5 shows the test of one of the struts and the method of measurements. 


\section{RESULTS OF STRUT TESTS.}

The number assigned to the struts tested in this investigation, together with the length, $L / r$ ratio, kind of test, the transverse load, and properties, are given in Table 4. The dimensions of each strut tested were determined from a large number of micrometer measurements of wall thickness made at each end. Measurements of the end sections showed that while the wall thickness was quite variable (see Table 4) it was practically the same for corresponding points at the two ends of a tube, so that the end measurements fairly represent the wall thickness throughout the length of a strut.

The ultimate loads of the columns and of the struts with transverse loading are given in Table 5. The table also includes the effect of the transverse loading; that is, the bending stress, the computed and measured deflection at the center of the strut produced by the transverse load alone.

The stress-deflection curves for the struts are shown in Figures 6 to 9 , inclusive. The effect of increasing the transverse loading in decreasing the column strength and increasing the rate of deflection are shown in these curves.

TABLE 4.-Outline of Tests and Properties of Struts.

11/2 INCBES, 20 GAUGE.

\begin{tabular}{|c|c|c|c|c|c|c|c|c|}
\hline Strut number. ${ }^{1}$ & Length. & $\underset{r a t i o .}{\frac{L}{r}}$ & Kind of test. & $\begin{array}{c}\text { Trans- } \\
\text { verse } \\
\text { load. } \\
\text { w }\end{array}$ & $\begin{array}{l}\text { Maximum } \\
\text { and mini- } \\
\text { mum wall } \\
\text { thickness } \\
\text { (average of } \\
\text { both ends). }\end{array}$ & $\begin{array}{c}\text { Area. } \\
\boldsymbol{A}\end{array}$ & $\begin{array}{c}\text { Moment } \\
\text { of } \\
\text { Inertia. } \\
I\end{array}$ & $\begin{array}{c}\text { Distance } \\
\text { to com- } \\
\text { pressive } \\
\text { extreme } \\
\text { fiber. } \\
c\end{array}$ \\
\hline 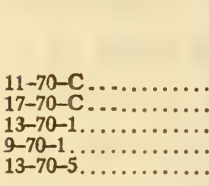 & $\begin{array}{r}\text { Inches. } \\
36.3 \\
36.3 \\
36.2 \\
36.2 \\
36.3\end{array}$ & $\begin{array}{l}70 \\
70 \\
70 \\
70 \\
70\end{array}$ & $\begin{array}{l}\text { Column } \ldots . . . \\
\ldots \ldots \text { do } . . . . . \\
\text { Combine.... } \\
\ldots \ldots \text { do } \ldots . . . .\end{array}$ & $\begin{array}{c}\begin{array}{c}\text { Lbs. per } \\
\text { linear } \\
\text { inch. }\end{array} \\
\ldots \ldots \ldots \\
\cdots \\
1.25 \\
1.25 \\
5.0\end{array}$ & $\begin{array}{c}\text { Lnch. } \\
0.035-0.0335 \\
.0365-.0355 \\
.034-.036 \\
.034-.035 \\
.033-.037\end{array}$ & 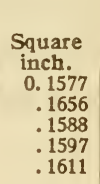 & $\begin{array}{l}\text { In. } \\
0.0424 \\
.0444 \\
.0427 \\
.0429 \\
.0432\end{array}$ & $\begin{array}{c}\text { Inch. } \\
0.743 \\
.745 \\
.75 \\
.755 \\
.770\end{array}$ \\
\hline 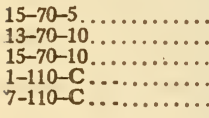 & $\begin{array}{l}36.3 \\
36.2 \\
36.2 \\
57 \\
57\end{array}$ & $\begin{array}{r}70 \\
70 \\
70 \\
110 \\
110\end{array}$ & 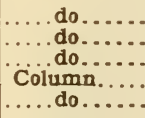 & $\begin{array}{l}5.0 \\
10.0 \\
10.0 \\
\ldots \ldots \ldots\end{array}$ & $\begin{array}{l}.0325-.035 \\
.033-.036 \\
.034-.0337 \\
.034-.038 \\
.032-.036\end{array}$ & $\begin{array}{l}.1552 \\
.1579 \\
.1556 \\
.1656 \\
.1566\end{array}$ & $\begin{array}{l}.0418 \\
.0424 \\
.0419 \\
.0444 \\
.0421\end{array}$ & $\begin{array}{l}.764 \\
.763 \\
.749 \\
.75 \\
.75\end{array}$ \\
\hline 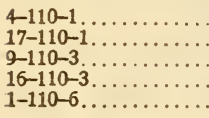 & $\begin{array}{l}57 \\
57 \\
57 \\
57 \\
57\end{array}$ & $\begin{array}{l}110 \\
110 \\
110 \\
110 \\
110\end{array}$ & $\begin{array}{l}\text { Combine.... } \\
\ldots \ldots \text { do } \ldots . . . \\
\ldots \ldots \text { do } \ldots . . \\
\ldots \ldots \text { do } \ldots . . . \\
\ldots \ldots\end{array}$ & $\begin{array}{l}1.0 \\
1.0 \\
3.0 \\
3.0 \\
6.05\end{array}$ & $\begin{array}{l}.035-.036 \\
.036-.035 \\
.0355-.036 \\
.035-.0375 \\
.0393-.0337\end{array}$ & $\begin{array}{l}.1633 \\
.1633 \\
.1647 \\
.1667 \\
.1678\end{array}$ & $\begin{array}{l}.0438 \\
.0438 \\
.0442 \\
.0447 \\
.0450\end{array}$ & $\begin{array}{l}.755 \\
.745 \\
.752 \\
.763 \\
.723\end{array}$ \\
\hline 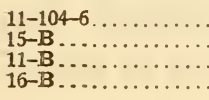 & $\begin{array}{l}53.8 \\
36 \\
36 \\
36\end{array}$ & $\begin{array}{r}104 \\
\ldots \ldots\end{array}$ & $\begin{array}{l}\text { Bend } \\
\ldots \ldots \text { do... } \\
\ldots \text { do... }\end{array}$ & 6.05 & 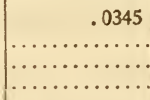 & $\begin{array}{r}.1588 \\
\ldots \ldots \ldots\end{array}$ & $\begin{array}{c}.0427 \\
\ldots \ldots \ldots \ldots \\
\ldots \ldots \ldots\end{array}$ & \begin{tabular}{c}
\multicolumn{1}{c}{.75} \\
$\cdots \cdots \cdots \cdots$ \\
$\cdots \cdots \cdots \cdots$ \\
$\cdots \cdots \cdots$
\end{tabular} \\
\hline
\end{tabular}

1 First number denotes the section of tubing from which the strut was cut, second number denotes the $L / r$ ratio, and the third number the transverse load. 


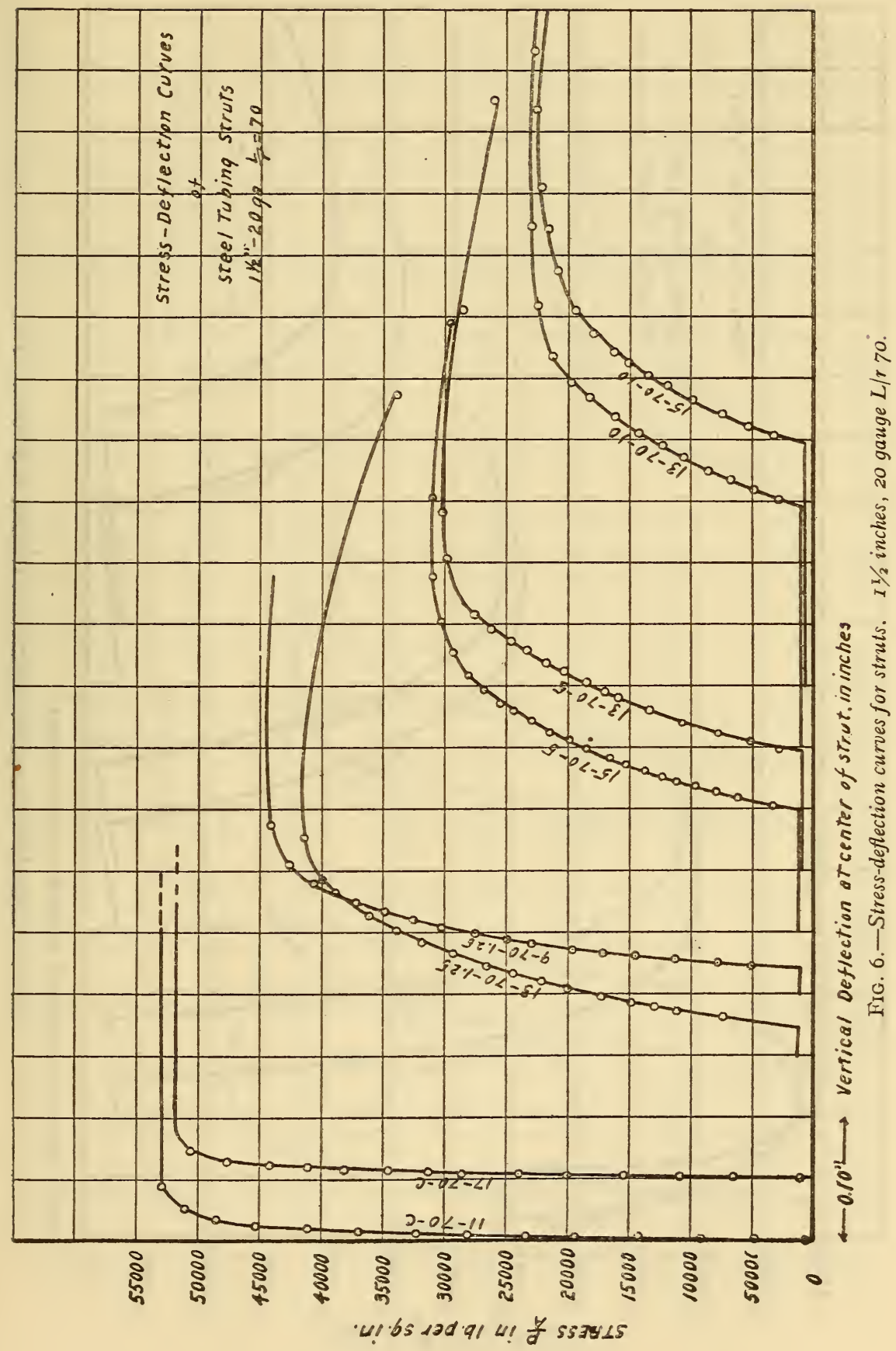




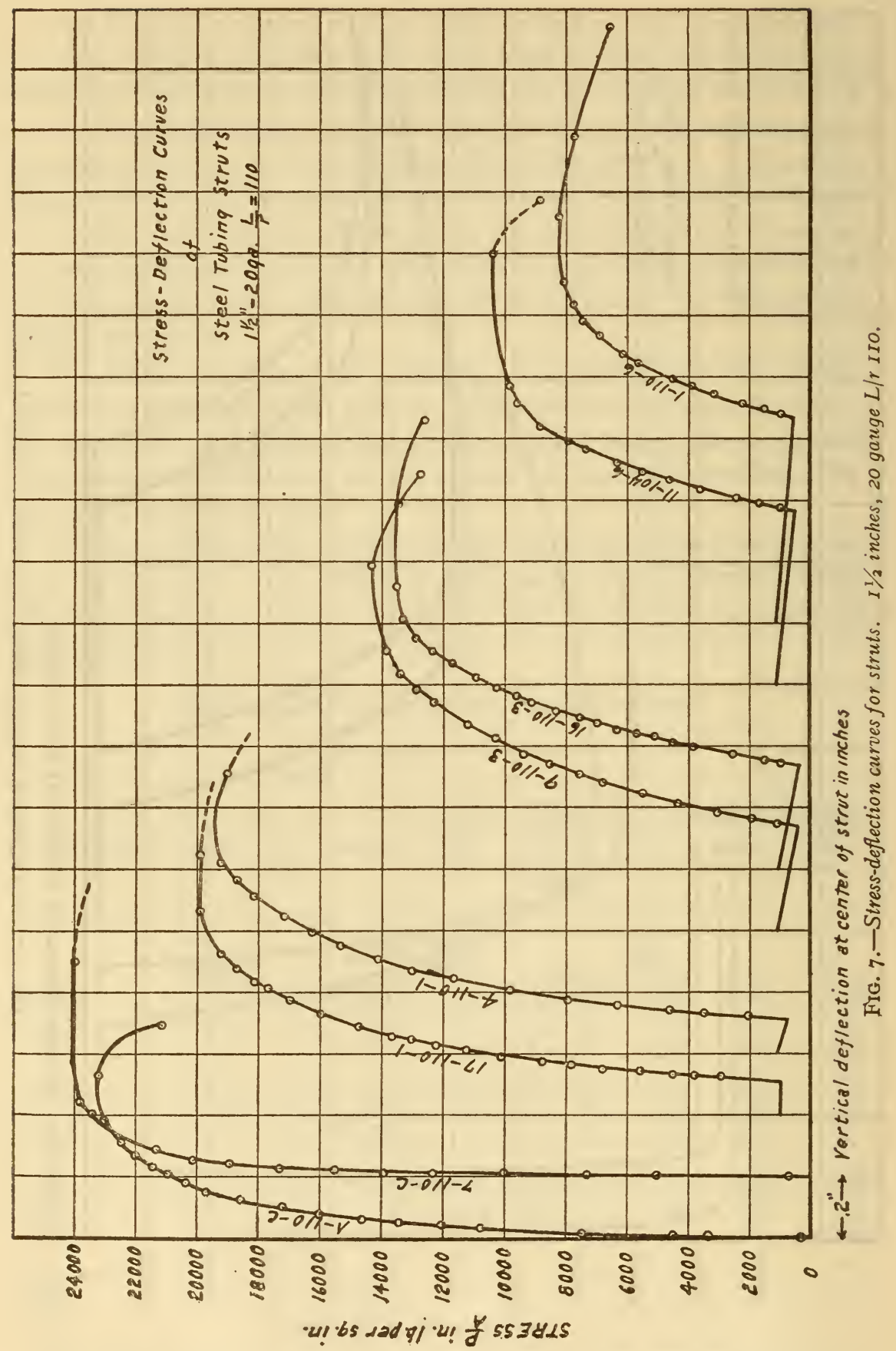




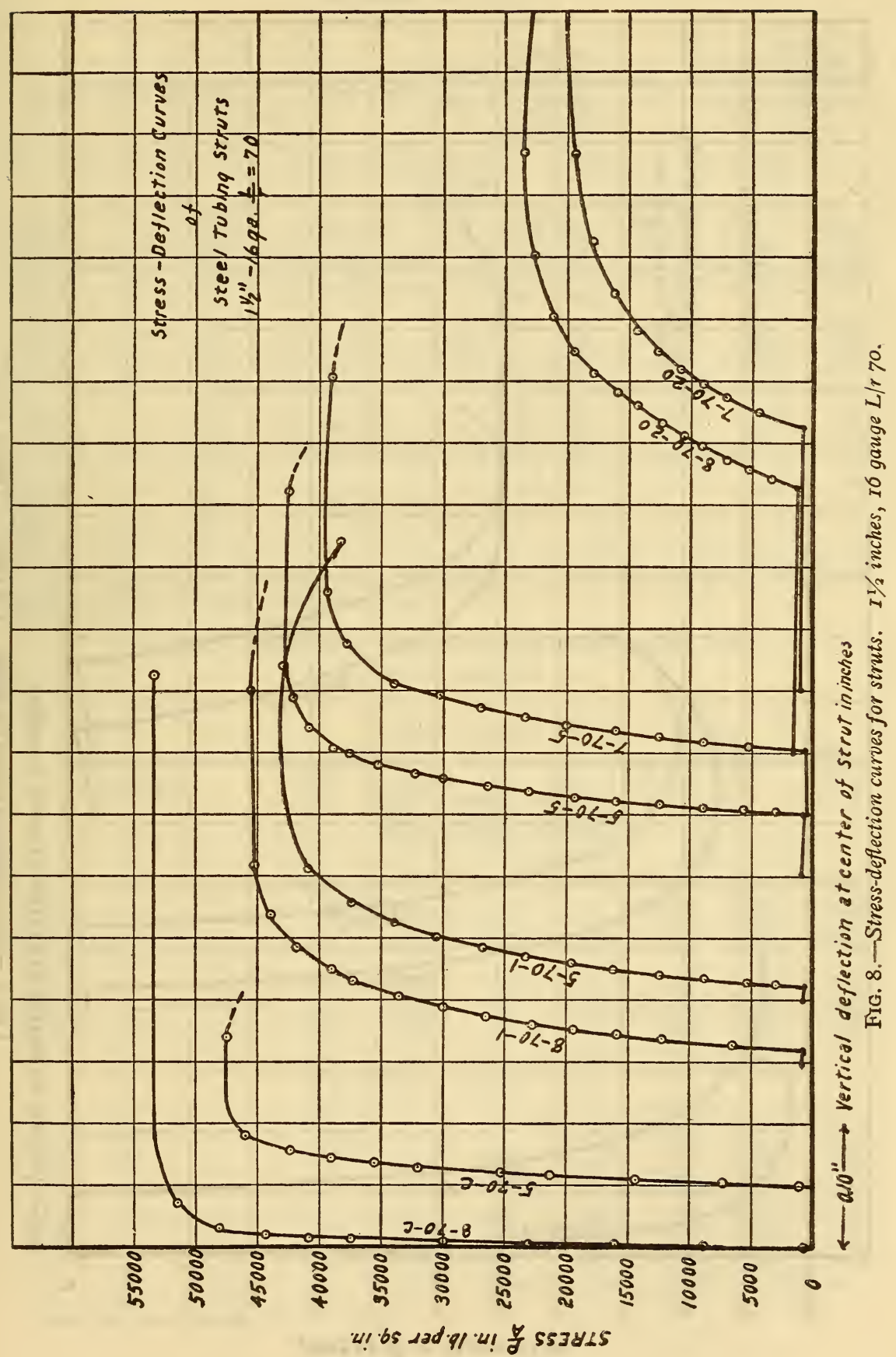




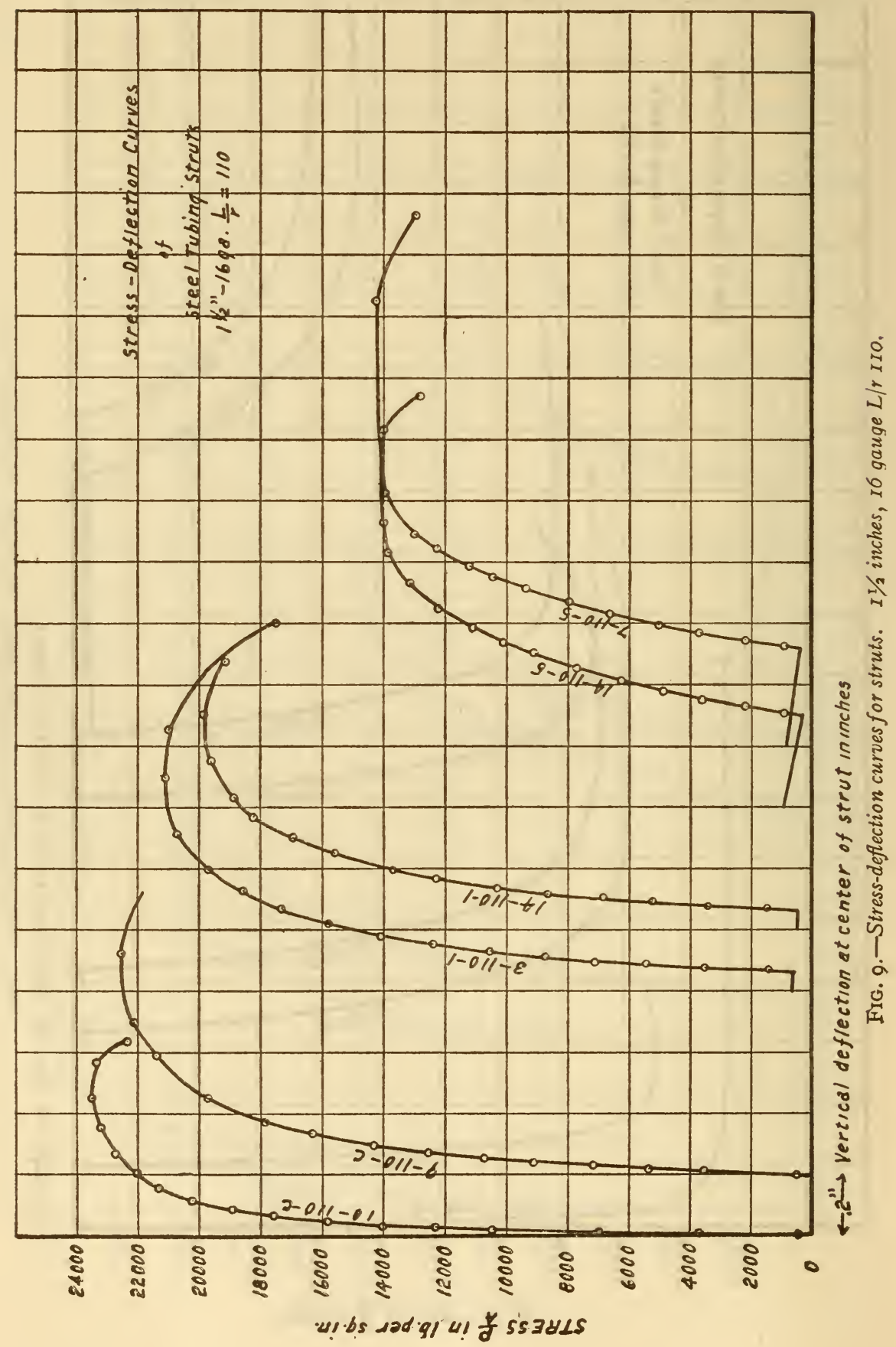


TABLE 4.-Outline of Tests and Properties of Struts-Continued.

11/2 INCHES, 16 GAUGE.

\begin{tabular}{|c|c|c|c|c|c|c|c|c|}
\hline Strut number.1 & Length. & $\frac{L}{r}$ & Kind of test. & $\begin{array}{c}\text { Trans- } \\
\text { verse } \\
\text { load. } \\
w\end{array}$ & $\begin{array}{l}\text { Maximum } \\
\text { and mini- } \\
\text { mum wail } \\
\text { thickness } \\
\text { (average of } \\
\text { both ends). }\end{array}$ & $\begin{array}{c}\text { Area, } \\
A\end{array}$ & $\begin{array}{l}\text { Moment } \\
\text { of } \\
\text { inertia. } \\
I\end{array}$ & $\begin{array}{l}\text { Distance } \\
\text { to com- } \\
\text { pressive } \\
\text { extreme } \\
\text { fiber. } \\
c\end{array}$ \\
\hline 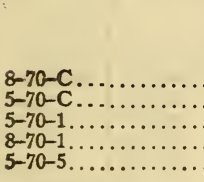 & $\begin{array}{r}\text { Inches. } \\
35.7 \\
35.7 \\
35.7 \\
35.7 \\
35.7\end{array}$ & $\begin{array}{l}70 \\
70 \\
70 \\
70 \\
70\end{array}$ & $\begin{array}{l}\text { Column } \ldots . . . \\
\ldots \ldots \text { do } \ldots . . . . \\
\text { Combine.... } \\
\ldots \ldots \text { do......... }\end{array}$ & 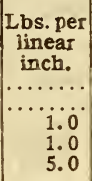 & $\begin{array}{l}\text { Irch. } \\
0.0635-0.0615 \\
.0055-.0605 \\
.060-.065 \\
.0523-.063 \\
.066-.060\end{array}$ & $\begin{array}{c}\text { Square } \\
\text { inch. } \\
0.2822 \\
.2832 \\
.2822 \\
.2844 \\
.2844\end{array}$ & $\begin{array}{l}\text { In. } \\
0.073 \\
.0735 \\
.0730 \\
.0735 \\
.0735\end{array}$ & $\begin{array}{r}\text { Inch. } \\
0.744 \\
.739 \\
.763 \\
.755 \\
.734\end{array}$ \\
\hline $\begin{array}{l}7-70-5 \ldots \ldots \ldots \ldots \ldots \\
8-70-20 \ldots \ldots \ldots \ldots \ldots \\
7-70-20 \ldots \ldots \ldots \ldots \\
10-110-C \ldots \ldots \ldots\end{array}$ & $\begin{array}{l}35.7 \\
35.7 \\
35.7 \\
56.2\end{array}$ & $\begin{array}{r}70 \\
70 \\
70 \\
110\end{array}$ & $\begin{array}{l}\ldots . . \text { do } \ldots . . . \\
\ldots \ldots \text { do } \ldots . . . . \\
\ldots \text { do } \ldots . . . \\
\text { Column }\end{array}$ & $\begin{array}{r}5.0 \\
20.0 \\
20.0\end{array}$ & $\begin{array}{l}.064-.060 \\
.059-.063 \\
.062-.065\end{array}$ & $\begin{array}{l}.2801 \\
.2846 \\
.2803 \\
.287\end{array}$ & $\begin{array}{l}.0725 \\
.0735 \\
.0725 \\
.074\end{array}$ & $\begin{array}{l}.740 \\
.74 \\
.766 \\
.75\end{array}$ \\
\hline $\begin{array}{l}9-110-C \ldots \ldots \ldots \\
3-110-1, \ldots \ldots \ldots \ldots \\
14-110-1 \ldots \ldots \ldots \ldots \\
14-110-5 \ldots \ldots \ldots \ldots\end{array}$ & $\begin{array}{l}56.1 \\
56.1 \\
56.1 \\
56.1\end{array}$ & $\begin{array}{l}110 \\
110 \\
110 \\
110\end{array}$ & 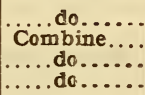 & $\begin{array}{r}1.0 \\
1.0 \\
5.0\end{array}$ & $\begin{array}{l}.058-.064 \\
.0655-.061 \\
.0615-.0685 \\
.062-.056\end{array}$ & $\begin{array}{l}.2803 \\
.2855 \\
.2920 \\
.2887\end{array}$ & $\begin{array}{l}.0725 \\
.0738 \\
.0754 \\
.0745\end{array}$ & $\begin{array}{l}.75 \\
.738 \\
.767 \\
.762\end{array}$ \\
\hline $\begin{array}{l}7-110-5 \ldots \ldots \ldots \ldots \\
5-B \\
8-B \ldots \ldots \ldots \ldots \ldots \\
10-B \ldots \ldots \ldots \ldots\end{array}$ & $\begin{array}{l}56.1 \\
36 \\
36 \\
36\end{array}$ & 110 & Bend $\ldots \ldots$ & 5.0 & $\begin{array}{c}.063-.060 \\
\ldots \ldots \ldots \ldots \ldots\end{array}$ & .2780 & .0720 & $\begin{array}{l}.742 \\
\cdots \cdots \\
\cdots \cdots\end{array}$ \\
\hline
\end{tabular}

1 First number denotes the section of tubing from which the strut was cut, second number denotes the $L / r$ ratio, and the third number the transverse load.

TABLE 5.-Results of Column and Combined Loading Tests.

$11 / 2$ INCHES, 20 GAUGE $\frac{L}{r}=70$.

\begin{tabular}{|c|c|c|c|c|c|c|c|}
\hline \multirow{2}{*}{ Strut number. } & \multirow{2}{*}{$\begin{array}{l}\text { Trans- } \\
\text { verse } \\
\text { load. }\end{array}$} & \multicolumn{3}{|c|}{ Effect of transverse load. } & \multirow{2}{*}{$\begin{array}{l}\text { Ultimate } \\
\text { end load. } \\
\qquad P\end{array}$} & \multirow{2}{*}{$\begin{array}{l}\text { Com- } \\
\text { pressive } \\
\text { stress. } \\
\frac{P}{A}\end{array}$} & \multirow{2}{*}{$\begin{array}{l}\text { Euler } \\
\text { load. } \\
P_{E}=\frac{\pi^{2} E J}{L^{8}}\end{array}$} \\
\hline & & $\begin{array}{l}\text { Bending, } \\
\text { stress at } \\
\text { extreme } \\
\text { fiber. }\end{array}$ & $\begin{array}{l}\text { Com- } \\
\text { puted de- } \\
\text { flection } \\
\text { at center.2 }\end{array}$ & $\begin{array}{l}\text { Meas- } \\
\text { ured de- } \\
\text { flection } \\
\text { at center. }\end{array}$ & & & \\
\hline $\begin{array}{l}11-70-C . . \\
17-70-C . . . \\
13-70-1 \ldots \\
9-70-1 \ldots\end{array}$ & $\begin{array}{r}\text { Pounds. } \\
\text { Col. } \\
\text { Col. } \\
1.25 \\
1.25\end{array}$ & $\begin{array}{r}\text { Lbs./in.2 } \\
3,600 \\
3,600\end{array}$ & \begin{tabular}{|c|} 
Inch. \\
$\ldots .023$ \\
0.0215 \\
0.0. \\
\end{tabular} & $\begin{array}{r}\text { Inch. } \\
0.023 \\
.020\end{array}$ & $\begin{array}{r}\text { Pounds. } \\
8,340 \\
8,600 \\
6,540 \\
7,100\end{array}$ & $\begin{array}{r}\text { Lbs./in.2 } \\
52,900 \\
51,900 \\
41,200 \\
44,500\end{array}$ & $\begin{array}{r}\text { Pounds. } \\
9,230 \\
9,665 \\
9,300 \\
9,370\end{array}$ \\
\hline $\begin{array}{l}13-70-5 \\
15-70-5, \ldots \ldots \\
13-70-10 \ldots \ldots \\
15-70-10 \ldots \ldots\end{array}$ & $\begin{array}{r}5.0 \\
5.0 \\
10.0 \\
10.0\end{array}$ & $\begin{array}{l}14,600 \\
14,950 \\
29,400 \\
29,200\end{array}$ & $\begin{array}{l}.090 \\
.093 \\
.183 \\
.196\end{array}$ & $\begin{array}{l}.097 \\
.099 \\
.192 \\
.198\end{array}$ & $\begin{array}{l}4,850 \\
4,800 \\
3,620 \\
3,500\end{array}$ & $\begin{array}{l}30,100 \\
30,900 \\
22,900 \\
22,500\end{array}$ & $\begin{array}{l}9,435 \\
9,130 \\
9,261 \\
9,151\end{array}$ \\
\hline
\end{tabular}

$11 / 2$ INCHES, 20 GAUGE $\frac{L}{r}=110$.

\begin{tabular}{|c|c|c|c|c|c|c|c|}
\hline 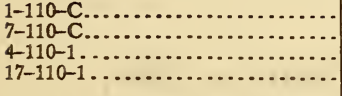 & $\begin{array}{l}\text { Col. } \\
\text { Col. } \\
1.0 \\
1.0\end{array}$ & $\begin{array}{l}7,000 \\
6,900\end{array}$ & $\begin{array}{c}0.1085 \\
.108\end{array}$ & $\begin{array}{r}0.113 \\
.111\end{array}$ & $\begin{array}{l}3,840 \\
3,770 \\
3,170 \\
3,250\end{array}$ & $\begin{array}{l}23,200 \\
24,000 \\
19,400 \\
19,900\end{array}$ & $\begin{array}{l}3,910 \\
3,710 \\
3,858 \\
3,858\end{array}$ \\
\hline 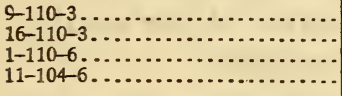 & $\begin{array}{l}3.0 \\
3.0 \\
6.05 \\
6.05\end{array}$ & $\begin{array}{l}20,700 \\
20,800 \\
39,500 \\
38,100\end{array}$ & $\begin{array}{l}.322 \\
.318 \\
.638 \\
.534\end{array}$ & $\begin{array}{l}.340 \\
.340 \\
.666 \\
.566\end{array}$ & $\begin{array}{l}2,360 \\
2,260 \\
1,380 \\
1,640\end{array}$ & $\begin{array}{r}14,300 \\
13,550 \\
8,200 \\
10,300\end{array}$ & $\begin{array}{l}3,894 \\
3,938 \\
3,964 \\
4,222\end{array}$ \\
\hline
\end{tabular}

${ }_{1}^{1}$ Bending stress at extreme fiber $S_{B}=\frac{x}{8} \frac{w L^{2}}{\frac{I}{c}}$.

2 Deflection at center of strut $l_{\mathrm{B}}=\frac{5}{38_{4}} \frac{w L^{4}}{E I}$. 
TABLE 5.-Results of Column and Combined Loading Tests-Continued.

$11 / 2$ INCHES, 16 GAUGE $\frac{L}{r}=70$.

\begin{tabular}{|c|c|c|c|c|c|c|c|}
\hline \multirow[b]{2}{*}{ Strut number. } & \multirow[b]{2}{*}{$\begin{array}{l}\text { Trans- } \\
\text { verse } \\
\text { load. }\end{array}$} & \multicolumn{3}{|c|}{ Effect of transverse load. } & \multirow[b]{2}{*}{$\begin{array}{c}\text { Ultimate } \\
\text { end load. } \\
P\end{array}$} & \multirow{2}{*}{$\begin{array}{l}\text { Com- } \\
\text { pressive } \\
\text { stress. } \\
\frac{P}{A}\end{array}$} & \multirow{2}{*}{$\begin{array}{c}\text { Euler } \\
\text { load. } \\
P_{\mathrm{a}=}=\frac{\pi^{2} E I}{L^{2}}\end{array}$} \\
\hline & & $\begin{array}{l}\text { Bending, } \\
\text { stress at } \\
\text { extreme } \\
\text { fiber.1 }\end{array}$ & $\begin{array}{c}\text { Com- } \\
\text { puted de- } \\
\text { flection } \\
\text { at center. }\end{array}$ & $\begin{array}{l}\text { Meas- } \\
\text { ured de- } \\
\text { fection } \\
\text { at center. }\end{array}$ & & & \\
\hline 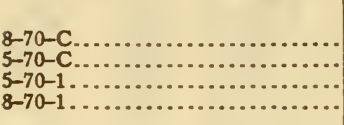 & $\begin{array}{r}\text { Pounds. } \\
\text { Col. } \\
\text { Col. } \\
1.0 \\
1.0\end{array}$ & \begin{tabular}{r} 
Lbs./in. \\
\hdashline... \\
\hdashline \\
1,660 \\
1,630
\end{tabular} & $\begin{array}{c}\text { Inch. } \\
\cdots \\
0.010 \\
.010\end{array}$ & $\begin{array}{r}\text { Inch. } \\
\ldots .010 \\
.010\end{array}$ & $\begin{array}{r}\text { Pounds. } \\
14,900 \\
13,500 \\
12,100 \\
12,950\end{array}$ & $\begin{array}{r}\text { Lbs./in. } \\
52,800 \\
47,650 \\
42,900 \\
45,500\end{array}$ & $\begin{array}{r}\text { Pounds. } \\
16,390 \\
16,510 \\
16,390 \\
16,510\end{array}$ \\
\hline $\begin{array}{l}5-70-5 \\
7-70-5 \\
8-70-20 \\
7-70-20 \\
7\end{array}$ & $\begin{array}{r}5.0 \\
5.0 \\
20.0 \\
20.0\end{array}$ & $\begin{array}{r}7,900 \\
8,100 \\
32,500 \\
33,700\end{array}$ & $\begin{array}{l}.049 \\
.050 \\
.199 \\
.201\end{array}$ & $\begin{array}{l}.051 \\
.053 \\
.214 \\
.213\end{array}$ & $\begin{array}{r}12,100 \\
11,000 \\
6,620 \\
5,540\end{array}$ & $\begin{array}{l}42,500 \\
39,300 \\
23,250 \\
19,780\end{array}$ & $\begin{array}{l}16,510 \\
16,280 \\
16,510 \\
16,280\end{array}$ \\
\hline
\end{tabular}

$11 / 2$ INCHES, 16 GAUGE $\frac{L}{r}=110$.

\begin{tabular}{|c|c|c|c|c|c|c|c|}
\hline $\begin{array}{l}10-110-C \ldots \ldots \\
9-110-C . \ldots \ldots \\
3-110-1 \ldots \ldots \ldots\end{array}$ & $\begin{array}{l}\text { Col. } \\
\text { Col. } \\
1.0\end{array}$ & $\ldots \ldots$ & $\begin{array}{c}\cdots \\
\cdots\end{array}$ & $\begin{array}{c}\cdots \\
\cdots \\
\cdots\end{array}$ & $\begin{array}{l}6,740 \\
6,300 \\
6,020\end{array}$ & $\begin{array}{l}23,500 \\
22,500 \\
21,100\end{array}$ & $\begin{array}{l}6,730 \\
6,593 \\
6,715\end{array}$ \\
\hline $\begin{array}{l}14-110-1 \\
14-110-5 . \\
7-110-5 . \ldots .\end{array}$ & $\begin{array}{l}1.0 \\
5.0 \\
5.0\end{array}$ & $\begin{array}{r}4,000 \\
10,100 \\
20,300\end{array}$ & $\begin{array}{l}.059 \\
.299 \\
.309\end{array}$ & $\begin{array}{l}.062 \\
.302 \\
.318\end{array}$ & $\begin{array}{l}5,800 \\
4,050 \\
3,960\end{array}$ & $\begin{array}{l}19,870 \\
14,050 \\
14,250\end{array}$ & $\begin{array}{l}6,855 \\
6,775 \\
6,548\end{array}$ \\
\hline
\end{tabular}

1 Bending stress at extreme fiber $S_{B}=\frac{I w L^{3}}{8 \frac{l}{c}}$.

Deffection at center of strut $l_{B}=\frac{5}{3^{8} 4} \frac{w l^{4}}{E l}$.

\section{THEORY AND APPLICATION OF FORMULAS.}

\section{APPLICATION OF FORMULAS FOR COMBINED LOADING.}

The theory and generally applied formulas for determining the strength of struts subjected to combined axial and transverse loading are given by the following equations. The ultimate strength of the strut is reached when the maximum compressive stress at the extreme fiber

$$
f_{\mathrm{c}}=\frac{M_{\mathrm{o}}}{\frac{I}{C}}+\frac{P}{A} \text { approximates the yield point of the material }
$$

where

$f_{\mathrm{c}}=$ maximum compressive stress at the extreme fiber,

$M_{\mathrm{o}}=$ maximum bending moment at the center of the strut,

$I=$ moment of inertia of the strut cross section,

$c=$ distance of extreme fiber in compression from the neutral axis,

$P=$ ultimate end load,

$A=$ area of cross section of the strut. 
In the above equation $\frac{M_{\mathrm{o}}}{\frac{I}{C}}$ is the maximum bending stress in compression $S_{\mathrm{B}}$, and $\frac{P}{A}$ is the mean compressive stress on the section. The sum of these stresses is the maximum compressive stress acting on the strut, provided the stress remains proportional to the strain at these high stresses. It is to be expected that when this maximum intensity of compressive stress is about equal to the yield point of the material the strut will fail.

The value of $M_{\mathrm{o}}$ has been commonly computed by the rational formula ${ }^{1}$

$$
-M_{\mathrm{o}}=\frac{w E I}{P}\left(\sec \frac{\pi}{2} \sqrt{\frac{P}{P_{\mathrm{E}}}}-\mathrm{I}\right)
$$

or by Perry's approximate formula ${ }^{1}$

where

$$
-M_{\mathrm{o}}=\frac{\mathrm{I} w L^{2}}{8}\left(\frac{P_{\mathrm{E}}}{P_{\mathrm{E}}-P}\right)
$$

$w=$ uniform transverse load in pounds per linear inch,

$L=$ length of strut,

$E=$ modulus of elasticity,

$P=$ ultimate load,

$P_{\mathrm{E}}=\frac{\pi^{2} E I}{L^{2}}$, Euler's limiting value for ideal column.

If different values of ratios $P / P_{\mathrm{E}}$ be substituted in the formulas (2) and (3) above, the results show that Perry's approximate formula agrees very closely with the exact formula for ratios of $P / P_{\mathrm{E}}$ up to 0.75 .

The results obtained by application of the experimental data to the above formulas are given in Table 6. The table shows that the exact formula for computing the bending moment and Perry's approximate formula give practically identical results.

1 "Morley's Strength of Materials, I916," p. 282. In this edition Perry's formula is incorrectly written. It should read, $-M_{0}=\mathrm{I} / 8 w L^{2}\left(\frac{P_{E}}{P_{E}-P}\right)$

$$
90893^{\circ}-24-3
$$


TABLE 6.-Results by Commonly Used Formulas for Combined Loading.

$1 \frac{1}{2}$ INCHES, 20 GAUGE $\frac{L}{r}=70$.

\begin{tabular}{|c|c|c|c|c|c|c|c|}
\hline \multirow{3}{*}{ Strut number. } & \multirow{3}{*}{$\begin{array}{l}\text { Trans- } \\
\text { verse } \\
\text { load. }\end{array}$} & \multicolumn{2}{|c|}{ Exact formula. } & \multicolumn{2}{|c|}{ Perry's formula. } & \multirow{3}{*}{$\begin{array}{c}\text { Ratio. } \\
\frac{S_{\mathrm{B}}}{f_{0}}\end{array}$} & \multirow{3}{*}{$\begin{array}{l}\text { Yield } \\
\text { point of } \\
\text { material } \\
\text { (tension). }\end{array}$} \\
\hline & & \multicolumn{4}{|c|}{ Maximum- } & & \\
\hline & & $\begin{array}{l}\text { Bending } \\
\text { stress. }{ }^{1} \\
S_{\mathrm{B}}\end{array}$ & $\begin{array}{c}\text { Com- } \\
\text { pressive } \\
\text { stress. } \\
f_{0}\end{array}$ & $\begin{array}{l}\text { Bending } \\
\text { stress. }^{3} \\
S_{B}\end{array}$ & $\begin{array}{c}\text { Com- } \\
\text { pressive } \\
\text { stress. } \\
f_{0}\end{array}$ & & \\
\hline 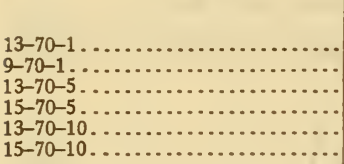 & $\begin{array}{r}\text { Pounds. } \\
1.25 \\
1.25 \\
5.0 \\
5.0 \\
10.0 \\
10.0\end{array}$ & $\begin{array}{r}\text { Lbs./in. }{ }^{2} \\
12,350 \\
15,100 \\
30,450 \\
32,000 \\
48,700 \\
48,300\end{array}$ & $\begin{array}{r}\text { Lbs./in. } .^{2} \\
53,550 \\
59,600 \\
60,550 \\
62,900 \\
71,600 \\
70,800\end{array}$ & $\begin{array}{r}\text { Lbs./in. }{ }^{2} \\
12,100 \\
14,900 \\
30,050 \\
31,500 \\
48,300 \\
47,400\end{array}$ & $\begin{array}{r}\text { Lbs./in.2 } \\
53,300 \\
59,400 \\
60,150 \\
62,400 \\
71,200 \\
69,900\end{array}$ & $\begin{array}{l}0.227 \\
.25 \\
.50 \\
.505 \\
.68 \\
.68\end{array}$ & $\begin{array}{r}\text { Lbs./in. }{ }^{2} \\
68,900 \\
66,800 \\
68,900 \\
69,900 \\
68,900 \\
69,900\end{array}$ \\
\hline
\end{tabular}

$11 / 2$ INCHES, 20 GAUGE $\frac{L}{r}=110$.

\begin{tabular}{|c|c|c|c|c|c|c|c|}
\hline 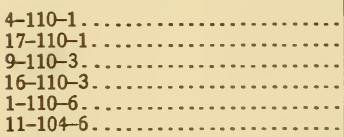 & $\begin{array}{l}1.0 \\
1.0 \\
3.0 \\
3.0 \\
6.05 \\
6.05\end{array}$ & $\begin{array}{l}39,600 \\
44,870 \\
53,300 \\
49,570 \\
61,160 \\
63,200\end{array}$ & $\begin{array}{l}59,100 \\
64,770 \\
67,600 \\
63,000 \\
69,360 \\
73,500\end{array}$ & $\begin{array}{l}39,300 \\
43,800 \\
52,700 \\
48,800 \\
60,600 \\
62,900\end{array}$ & $\begin{array}{l}58,700 \\
63,700 \\
67,000 \\
62,350 \\
68,800 \\
73,200\end{array}$ & $\begin{array}{r}0.67 \\
.68 \\
.78 \\
.78 \\
.88 \\
.86\end{array}$ & $\begin{array}{l}63,400 \\
67,400 \\
66,800 \\
62,200 \\
61,200 \\
69,000\end{array}$ \\
\hline
\end{tabular}

$11 / 2$ INCHES, 16 GAUGE $\frac{L}{r}=70$.

\begin{tabular}{|c|c|c|c|c|c|c|c|}
\hline $\begin{array}{l}5-70-1 . \ldots \ldots \\
8-70-1 . \ldots \ldots \\
5-70-5 \ldots \ldots \ldots \\
7-70-5 \ldots \ldots \ldots \\
8-70-20 \ldots \ldots \\
7-70-20 \ldots \ldots\end{array}$ & $\begin{array}{r}1.0 \\
1.0 \\
5.0 \\
5.0 \\
20.0 \\
20.0\end{array}$ & $\begin{array}{r}6,500 \\
7,740 \\
30,300 \\
25,600 \\
54,400 \\
51,900\end{array}$ & $\begin{array}{l}49,400 \\
53,200 \\
72,800 \\
64,900 \\
77650 \\
71,670\end{array}$ & $\begin{array}{r}6,400 \\
7,600 \\
29,500 \\
25,030 \\
54,200 \\
51,000\end{array}$ & $\begin{array}{l}49,300 \\
53,100 \\
72,000 \\
64,350 \\
77,450 \\
70,800\end{array}$ & $\begin{array}{r}0.13 \\
.14 \\
.41 \\
.39 \\
.70 \\
.72\end{array}$ & $\begin{array}{l}70,000 \\
70,200 \\
70,000 \\
67,500 \\
70,200 \\
67,500\end{array}$ \\
\hline
\end{tabular}

$1 \frac{1}{2}$ INCHES, 16 GAUGE $\frac{L}{r}=110$.

\begin{tabular}{|c|c|c|c|c|c|c|c|}
\hline 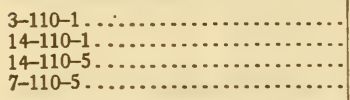 & $\begin{array}{l}1.0 \\
1.0 \\
5.0 \\
5.0\end{array}$ & $\begin{array}{l}38,300 \\
26,550 \\
50,940 \\
52,290\end{array}$ & $\begin{array}{l}59,400 \\
46,420 \\
65,000 \\
66,540\end{array}$ & $\begin{array}{l}38,000 \\
25,950 \\
50,100 \\
51,300\end{array}$ & $\begin{array}{l}59,100 \\
45,800 \\
64,150 \\
65,600\end{array}$ & $\begin{array}{r}0.64 \\
.57 \\
.78 \\
.78\end{array}$ & $\begin{array}{l}71,300 \\
64,600 \\
64,600 \\
67,500\end{array}$ \\
\hline
\end{tabular}

$$
\begin{aligned}
& { }^{1} S_{\mathrm{B}}=\frac{M_{\mathrm{O} C}}{I}=\frac{w E c}{P}\left(\sec \frac{\pi}{2} \sqrt{\frac{P}{P_{\mathrm{B}}}}-1\right) . \\
& { }^{2} f_{\mathrm{C}}=\frac{M_{\mathrm{O}}}{\frac{I}{c}}+\frac{P}{A} . \\
& { }^{3} S_{\mathrm{B}}=\frac{M_{\mathrm{O}} C}{I}=\frac{c}{I} \frac{w L^{2}}{8}\left(\frac{P_{\mathrm{E}}}{P_{\mathrm{E}}-P}\right) .
\end{aligned}
$$

A comparison of the maximum compressive stress values at failure, obtained by the commonly used formulas for struts with transverse loading with the yield point of the material, shows extreme inconsistency and wide variation. This variation is shown graphically in the left half of Figure ro. In this figure the maximum compressive stresses at failure computed by either of 
the commonly used formulas are plotted as ordinates. The plotted values represent either of the formulas shown on the figure, the difference between results obtained with each being too

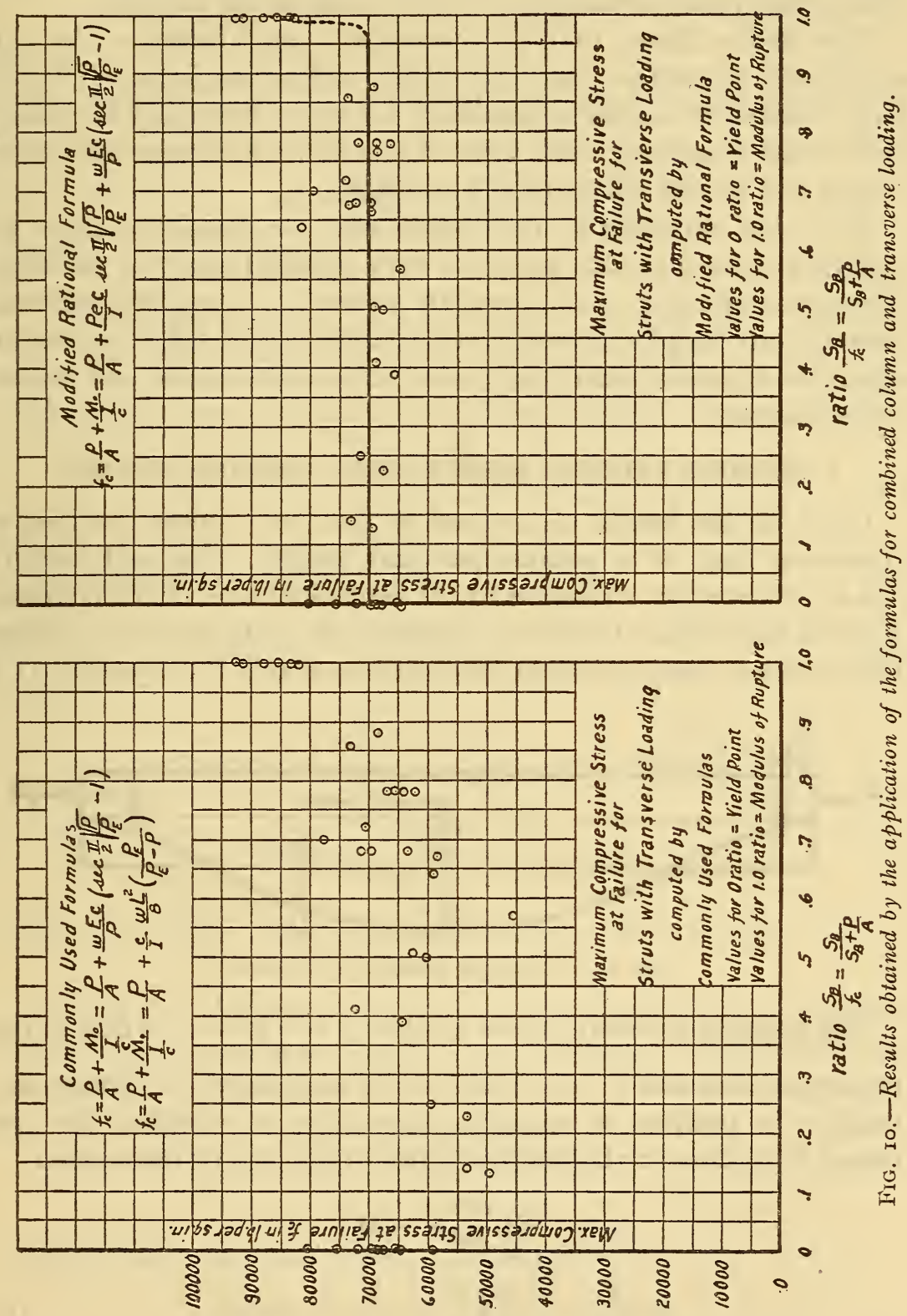

small to need separate designation. The ratios $\frac{S_{b}}{f_{c}}$ of bending stress to maximum compressive stress are plotted as abscissas, the ratio unity being the condition of pure transverse loading 
(modulus of rupture) and the axis of coordinate values o.o representing pure compression on short lengths. The maximum compressive stress (ordinate values) for the latter are practically the yield-point values obtained by tensile tests of the material.

This figure shows that the commonly used formulas do not accurately determine the load which will cause failure. For a large number of the tests, especially for short struts and for struts with small transverse load, these formulas give dangerously high values and should not be used for design.

The wide variation of the results and the possible danger in applying these formulas lead to a very detailed study of the conditions contributing to the strength of struts. A modified rational formula was found that will more accurately and safely represent stress conditions of struts subjected to combined axial and transverse loading.

\section{MODIFIED RATIONAL FORMULA FOR COMBINED LOADING.}

Let $L$ be the length of a round or free end column carrying a transverse load of $w$ pounds per unit length. The end load $P$ has an eccentricity $e$ relative to the centroidal axis of the column. Assume the origin $O$ midway between the ends, the line joining the centroids being taken as the coordinate axis $X$. (See fig. II.)

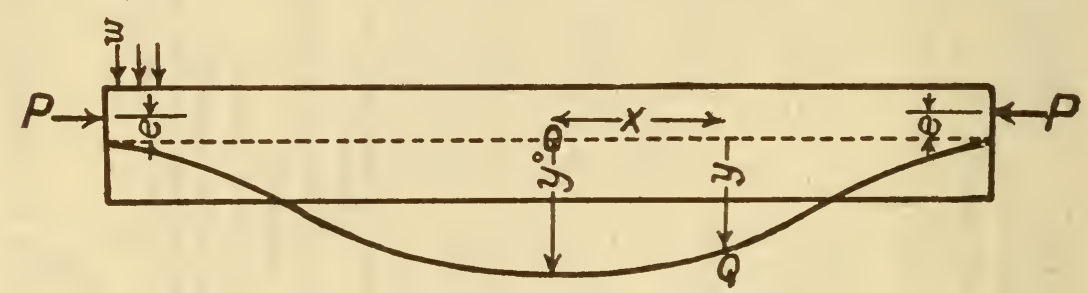

FIG. II.-Figure for derivation of formula.

The bending moment at any section $Q$ is $\frac{w}{2}\left(\frac{L^{2}}{4}-x^{2}\right)$ due to the transverse load and $P(y+e)$ due to the end thrust $P$. The eccentricity $e$ is positive or negative, depending on whether the end thrust $P$ is above or below the center of gravity of the section.

$$
\begin{gathered}
E I \frac{d^{2} y}{d x^{2}}=-M \\
E I \frac{d^{2} y}{d x^{2}}=-\frac{w}{2}\left(\frac{L^{2}}{4}-x^{2}\right)-P(y+e) \\
\frac{d^{2} y}{d x^{2}}+\frac{P y}{E I}=+\frac{w x^{2}}{2 E I}-\left(\frac{w L^{2}}{\delta E I}+\frac{P e}{E I}\right)
\end{gathered}
$$


The solution of this differential equation is

$$
y=\frac{w x^{2}}{2 P}-\frac{w L^{2}}{8 P}-\frac{w E I}{P^{2}}-e+A \cos \sqrt{\frac{P}{E I}} x+B \sin \sqrt{\frac{P}{E I}} x .
$$

The condition $\frac{d y}{d x}=O$ for $x=O$ and $y=O$ for $x=\frac{L}{2}$ gives $B=O$

hence,

$$
A=\left(\frac{w E I}{P^{2}}+e\right) \sec \frac{L}{2} \sqrt{\frac{P}{E I}}
$$

$$
y=\frac{w x^{2}}{2 P}-\frac{w L^{2}}{8 P}-\frac{w E I}{P^{2}}-8+\left[\left(\frac{w E I}{P^{2}}+e\right) \sec \frac{L}{2} \sqrt{\frac{P}{E I}} \cos \sqrt{\frac{P}{E I}} x\right]
$$

and at the origin or point of maximum moment where $x=0$

$$
y_{\mathrm{o}}=-\frac{w L^{2}}{8 P}-\left(\frac{W E I}{P^{2}}+e\right)\left(\mathrm{I}-\sec \frac{L}{2} \sqrt{\frac{P}{E I}}\right)
$$

The maximum moment at $O$ is

$$
\begin{aligned}
& -M_{\mathrm{o}}=P\left(y_{\mathrm{o}}+e\right)+\frac{\mathrm{I}}{8} w L^{2} \\
& -M_{\mathrm{o}}=P e-\left[\frac{w E I}{P}+P e\right]\left[\mathrm{I}-\sec \frac{L}{2} \sqrt{\frac{P}{E I}}\right] \\
& -M_{\mathrm{o}}=P e \sec \frac{\pi}{2} \sqrt{\frac{P}{P_{\mathrm{E}}}}+\frac{w E I}{P}\left(\sec \frac{\pi}{2} \sqrt{\frac{P}{P_{\mathrm{E}}}}-\mathrm{I}\right)
\end{aligned}
$$

where $P_{\mathrm{E}}=\frac{\pi^{2} E I}{L^{2}}$, Euler's limiting value for ideal column.

Therefore, to cause failure of a strut subjected to combined column and transverse loading, the maximum compressive stress at the extreme fiber is

$$
f_{\mathrm{c}}=\frac{M_{\mathrm{o}}}{\frac{I}{c}}+\frac{P}{A}
$$

approximates the yield point of the material, or

$$
f_{\mathrm{c}}=\frac{P}{A}+\frac{P e c}{I} \sec \frac{\pi}{2} \sqrt{\frac{P}{P_{\mathrm{E}}}}+\frac{w E c}{P}\left(\sec \frac{\pi}{2} \sqrt{\frac{P}{P_{\mathrm{E}}}}-\mathrm{I}\right)
$$

\footnotetext{
'See Church's Mechanics of Engineering, p. 382; r908.
} 
An examination of formula (4) for the bending moment shows that the first term takes account of the bending moment due to the eccentricity of the end load, and that the second term is the expression for a column with a transverse load combined with an axial end load. The formula also explains why the two commonly used formulas are undesirable for short struts and struts with small side loads. For short struts the end load $P$ is very large, so that for a given eccentricity the factor $P e \sec \frac{\pi}{2} \sqrt{\frac{P}{P_{\mathrm{E}}}}$ becomes a very large and important factor in computing the bending moment and consequently the bending stress. Also, when $w$ is small this factor is relatively large and important. On the other hand, when the struts are long $P$ is very small, making the factor $P e$ relatively less important. Likewise, when the transverse load $w$ is large, the second factor of the formula is large, and the eccentric factor has a relatively less influence on the strength of a strut.

\section{ECCENTRICITIES.}

(a) CAUSES OF ECCENTRICITY.

The modified rational formula indicates the importance of eccentricities. Such eccentricities are chiefly caused by the following conditions found in commercial tubing: (I) Deviation of the shape of the tubing from a circular section, (2) variation in wall thickness, and (3) deviation from straightness.

Measurements made of the external diameters of tubing used in the investigation indicated that the first condition-deviation from circular shape-is comparatively small, and this cause, consequently, was not considered. The other two conditions-variation in wall thickness and deviation from straightness-are perceptible to the eye, and the eccentricities resulting from these conditions proved to be important factors in determining the strength of struts. Figure 12 shows the variation in wall thickness of three of the struts tested.

(b) DETERMINATION OF ECCENTRICITY.

1. Eccentricity Due to Variation in Wall Thickness.Consider a cross section of the tube in which the wall thickness varies from an average minimum thickness of $t_{\mathrm{m} \text { n }}$ to an average maximum thickness of $t_{\mathrm{max}}$. (See fig. 13.) 


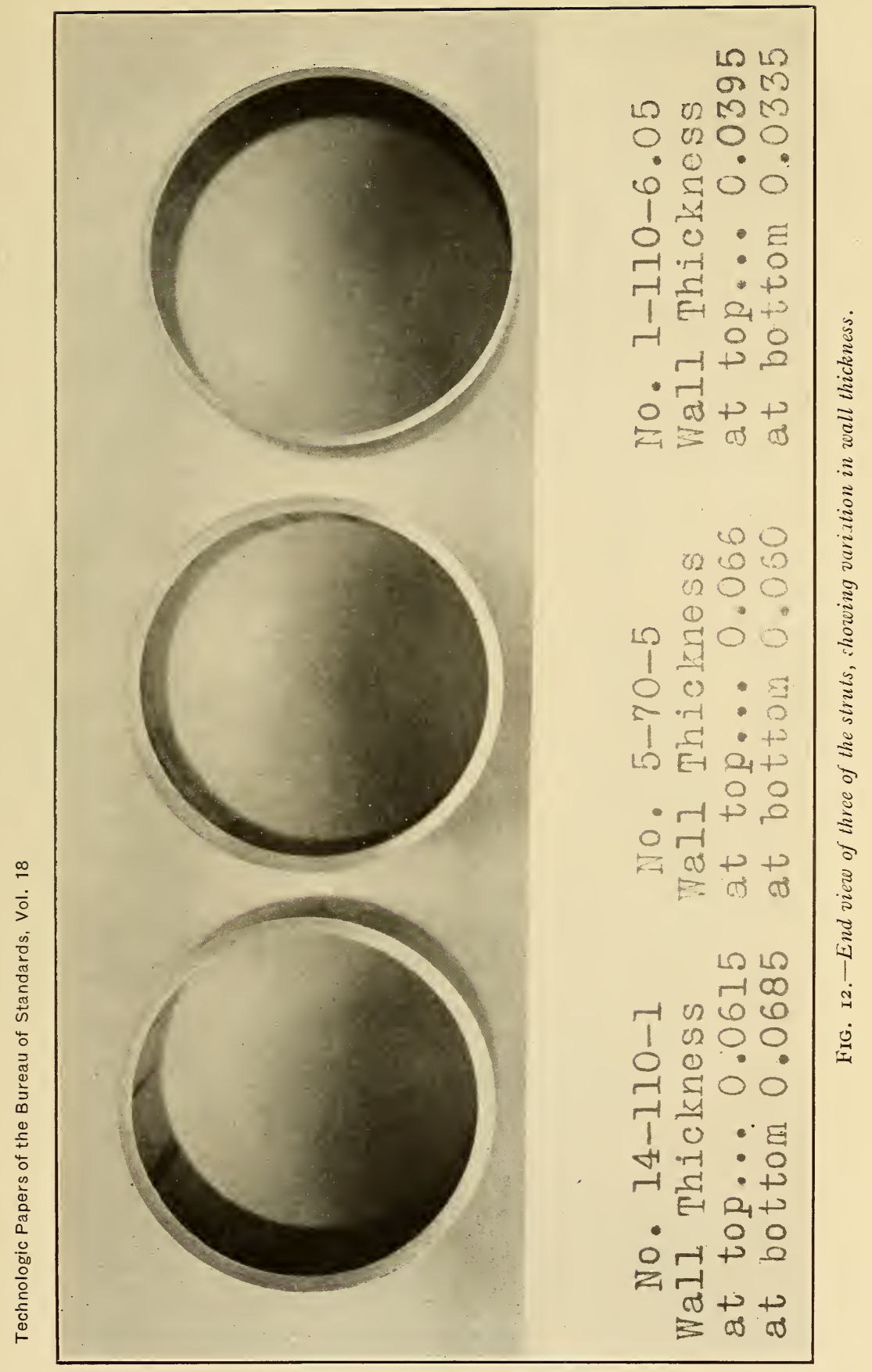



Let

$D=$ outside diameter of tube,

$k=$ distance from center of outer circle to center of inner circle,

$y=$ distance from center of outer circle to center of gravity of the section,

$k=\frac{t_{\max }-t_{\min }}{2}$

$y=\frac{A_{1}}{A_{0}-A_{1}} k$

where

$$
A_{0}=\frac{\pi D^{2}}{4} \text { and } A_{1}=\frac{\pi}{4}\left[D-\left(t_{\max }+t_{\min }\right)\right]^{2}
$$

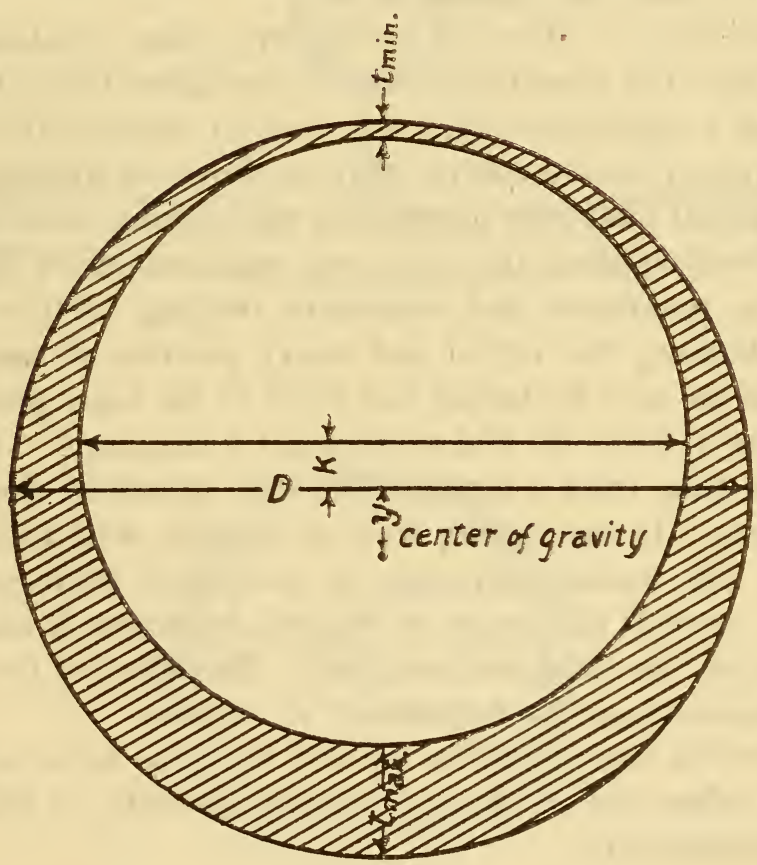

FIG. I3.-Method of determining eccentricity.

Section of tubing showing eccentricity due to variation in wall thickness.

In these tests the center of thrust was at the center of the inner circle, due to the fact that the lug on the hemispherical ball placed on the end of the strut filled the inside of the tubing.

Therefore the eccentricity $e_{\mathrm{w}}$ due to variation of wall thickness is

$e_{\mathrm{w}}=k+y=k\left(\mathrm{I}+\frac{A_{1}}{A_{0}-A_{1}}\right)=\frac{k A_{0}}{A_{0}-A_{1}}=\frac{k D^{2}}{2 D\left(t_{\max }+t_{\min }\right)-\left(t_{\max }+t_{\min }\right)^{2}}$ 
Should the minimum thickness be at a point other than at the top or bottom of the tube, the eccentricity about the horizontal axis is

$$
e_{\mathrm{w}}=(k+y) \sin \alpha=\frac{k A_{0}}{A_{0}-A_{1}} \sin \alpha
$$

where $\alpha$ is the angle that the diameter connecting the points of minimum and maximum wall thickness makes with the horizontal axis. In this investigation practically all of the specimens had the minimum thickness either at the top or bottom when tested. In the few cases where this condition did not exist the value of $\sin \alpha$ was considered as unity and the average thickness at the top and bottom used for $t_{\min }$ and $t_{\max }$ in the formula to determine the eccentricity about the horizontal axis.

2. Eccentricity Due to Deviation from Straightness.To determine the eccentricity due to deviation from straightness of the tube a micrometer dial was used to measure the deflection at the center of the column or strut, as shown in Figures 5 and I 4 . A small initial load was applied to the column, and before any side load was applied the tube was revolved in its ball-bearing ends. The maximum and minimum readings of the dial were noted, indicating the dotted and heavy position shown in Figure I4. In all the tests the tubing was tested in the lower position illustrated - that is, where the dial reading was a minimum. The deflection of the tube from a straight line was, therefore, downward in all the tests. If the tubing was of uniform wall thickness and diameter, the eccentricity due to deviation from straightness would be one-half the range of original deflection $R$ indicated by the dial when the strut was revolved. The effect of the variation of wall thickness can be determined as follows:

Consider the cross-sectional area of the tube to be as shown in Figure I 4 when the strut was concave upwards, as indicated by the micrometer dial.

Let

$R=$ difference in maximum and minimum deflection, as indicated by micrometer dial,

$e_{\mathrm{x}}=$ distance from center of thrust to center of inner circle.

$R=\frac{D}{2}+e_{\mathrm{x}}+k-\left[\frac{D}{2}-k-e_{\mathrm{x}}\right]=2 e_{\mathrm{x}}+2 k$

$\therefore e_{\mathrm{x}}=\frac{R-2 k}{2}$ the eccentricity due to deviation from straightness of the tube. 
The total eccentricity $e$-that is, the distance from the center of thrust to center of gravity of the section-is the sum of the eccentricities due to variation in wall thickness and deviation from straightness, or

$$
e=e_{\mathrm{w}}+e_{\mathrm{x}}=(k+y)+e_{\mathrm{x}}=k\left(\mathrm{I}+\frac{A_{1}}{A_{0}-A_{1}}\right)+\frac{R-2 k}{2}
$$

Should the maximum thickness of the wall $t_{\max }$ be up when the tube was tested, $k$ is negative and the eccentricity is

$$
e=-(k+y)+e_{\mathrm{x}}=-k\left(\mathrm{I}+\frac{A_{1}}{A_{0}-A_{1}}\right)+\frac{R+2 k}{2}
$$
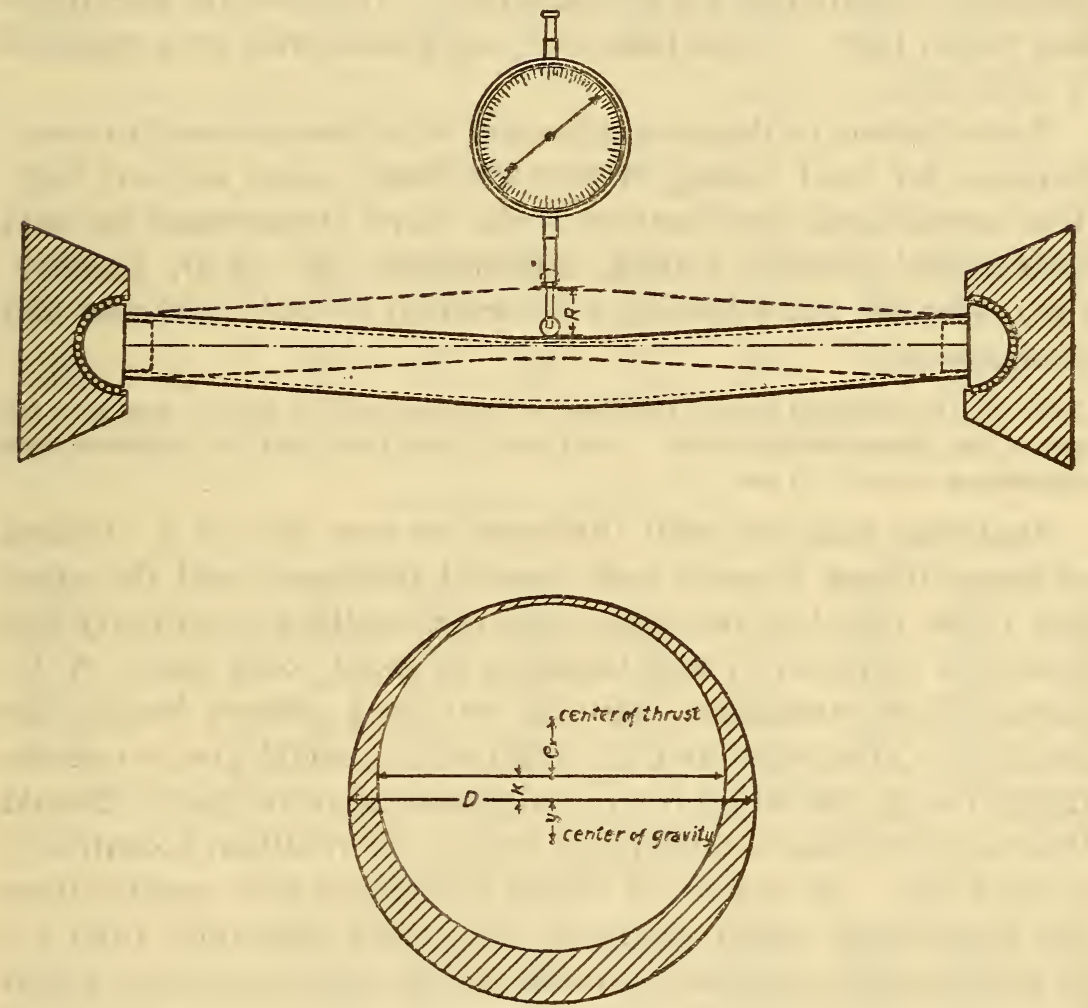

FIG. I4.-Method of determining eccentricity due to deviation from straightness.

(c) DISCUSSION.

Measurements of the eccentricities in the tubing showed that practically in every case the diameter connecting the minimum and maximum wall thickness was in the plane of deflection or warping of the tubing. Thus, the total effective eccentricity is either the sum or difference of the eccentricity due to variation in. 
wall thickness and eccentricity due to variation in straightness. This is to be expected, because any condition during the drawing of the tube that would cause the wall to be thin on one side and thick on the other would tend to warp the tube in the plane of symmetry; that is, the plane of minimum and maximum wall thickness. Moreover, any heat treatment that would relieve the unequal stresses in a tube of varying wall thickness would tend to produce warping in the plane of symmetry.

In Tables 7 and 8 are given the eccentricities resulting from wall variation and deviation from straightness, together with the total effective eccentricities for all the struts. The effective eccentricities varied from -0.008 inch to +0.039 inch, with an average of $+0.02 \mathrm{I}$ inch.

A comparison of these eccentricities with those allowed in specifications for steel tubing shows that these values are not high. The aeronautical specifications of the Navy Department for mild carbon-steel seamless tubing, Specification No. 58-B, January, 1920, specifies the following for variation in wall thickness and straightness:

Par. 8. The variation in wall thickness of the tubes may be plus or minus to per cent of the dimensions specified. In no part of any tube shall the departure from straightness exceed $\mathrm{x}$ in 600 .

Assuming that the wall thickness on one side of a $1 / 2$-inch r6-gauge tubing is 0.065 inch (normal thickness) and the other side ro per cent less, or 0.0585 inch, the resulting eccentricity due from this variation in wall thickness is about o.or 8 inch. A departure from straightness of $I$ in 600 for a 36 -inch length, the length of a strut with and $L / r$ ratio of 70 , would give an eccentricity due to deviation from straightness of 0.060 inch. Should these eccentricities be additive in a strut, the resultant eccentricity is 0.078 inch. In any lot of tubing complying with specifications the eccentricity would probably vary fairly uniformly from 0.0 to the extreme case above, so that the average eccentricity would be approximately $0.039 \mathrm{inch}$. This value is about twice the general average of $0.02 \mathrm{I}$ inch for the struts tested in this investigation. 
TABLE 7.-Eccentricities。

$11 / 2$ INCHES, 20 GAUGE $\frac{L}{r}=70$.

\begin{tabular}{|c|c|c|c|c|c|c|c|c|}
\hline \multirow{2}{*}{ Strut number. } & \multicolumn{2}{|c|}{$\begin{array}{l}\text { Average wall } \\
\text { thickness. }\end{array}$} & \multirow{2}{*}{$k^{1}$} & \multirow{2}{*}{$y^{2}$} & \multirow{2}{*}{$\begin{array}{c}\text { Eccen- } \\
\text { tricity } \\
\text { from } \\
\text { wall } \\
\text { variation. } \\
e_{\mathrm{rr}}=k+y\end{array}$} & \multirow{2}{*}{$\begin{array}{l}\text { Dial } \\
\text { deflec- } \\
\text { tion. } \\
R\end{array}$} & \multirow{2}{*}{$\begin{array}{l}\text { Eccen- } \\
\text { tricity } \\
\text { due to } \\
\text { crooked- } \\
\text { ness. } \\
e^{3}\end{array}$} & \multirow{2}{*}{$\begin{array}{l}\text { Total } \\
\text { eccen- } \\
\text { tricity. } \\
e=e_{\pi}+e_{x}\end{array}$} \\
\hline & $\begin{array}{l}\text { Top } \\
\text { side. }\end{array}$ & $\begin{array}{l}\text { Bottom } \\
\text { side. }\end{array}$ & & & & & & \\
\hline 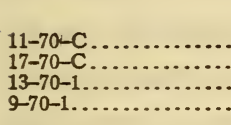 & $\begin{array}{l}\text { Inch. } \\
0.035 \\
.0365 \\
.0347 \\
.034\end{array}$ & $\begin{array}{l}\text { Inch. } \\
0.0335 \\
.0355 \\
.0347 \\
.0347\end{array}$ & $\begin{array}{l}\text { Inch. } \\
-0.0007 \\
-.0006 \\
.000 \\
+.0004\end{array}$ & $\begin{array}{l}\text { Inch. } \\
-0.007 \\
-.006 \\
.000 \\
+.0041\end{array}$ & $\begin{array}{l}\text { Inch. } \\
-0.0077 \\
-.0066 \\
.000 \\
+.0045\end{array}$ & $\begin{array}{r}\text { Iach. } \\
0.025 \\
.029 \\
.070 \\
.031\end{array}$ & $\begin{array}{l}\text { Inch. } \\
0.0132 \\
.015 \\
.035 \\
.015\end{array}$ & $\begin{array}{l}\text { Inch. } \\
0.0055 \\
.008 \\
.035 \\
.019\end{array}$ \\
\hline $\begin{array}{l}13-70-5 \ldots \ldots \ldots \ldots \\
15-70-5 \ldots \ldots \ldots \\
13-70-10 \ldots \ldots \ldots \\
15-70-10 \ldots \ldots \ldots\end{array}$ & $\begin{array}{l}.033 \\
.0325 \\
.033 \\
.0339\end{array}$ & $\begin{array}{l}.037 \\
.035 \\
.0355 \\
.0337\end{array}$ & $\begin{array}{l}+.0020 \\
+.0013 \\
+.0012 \\
-.0001\end{array}$ & $\begin{array}{l}+.020 \\
+.0135 \\
+.012 \\
-.001\end{array}$ & $\begin{array}{l}+.022 \\
+.0148 \\
+.0132 \\
-.0011\end{array}$ & $\begin{array}{l}.033 \\
.030 \\
.010 \\
.026\end{array}$ & $\begin{array}{l}.0145 \\
.0137 \\
.0035 \\
.013\end{array}$ & $\begin{array}{l}.036 \\
.0285 \\
.016 \\
.012\end{array}$ \\
\hline Average.. & & & & & +.0050 & & .0154 & .0200 \\
\hline
\end{tabular}

11/2 INCHES, 20 GAUGE $\frac{L}{r}=110$.

\begin{tabular}{|c|c|c|c|c|c|c|c|c|}
\hline 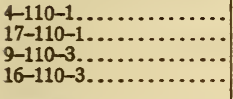 & $\begin{array}{l}0.035 \\
.036 \\
.0355 \\
.035\end{array}$ & $\begin{array}{l}0.036 \\
.035 \\
.036 \\
.0375\end{array}$ & $\begin{array}{r}+0.0005 \\
-.0005 \\
+.0002 \\
+.0013\end{array}$ & $\begin{array}{l}+0.005 \\
-.005 \\
+.002 \\
+.0125\end{array}$ & $\begin{array}{r}+0.0055 \\
-.0055 \\
+.0022 \\
+.0138\end{array}$ & $\begin{array}{l}0.044 \\
.031 \\
.068 \\
.034\end{array}$ & $\begin{array}{l}0.0215 \\
.016 \\
.034 \\
.0157\end{array}$ & $\begin{array}{l}0.027 \\
.011 \\
.036 \\
.0295\end{array}$ \\
\hline $\begin{array}{l}1-110-6 \ldots \ldots \ldots \ldots \ldots \\
11-104-6 \ldots \ldots \ldots\end{array}$ & $\begin{array}{l}.0393 \\
.0345\end{array}$ & $\begin{array}{l}.0337 \\
.0345\end{array}$ & $\begin{array}{c}-.0028 \\
.000\end{array}$ & $\begin{aligned}-.0267 \\
.000\end{aligned}$ & $\begin{aligned}-.0295 \\
.000\end{aligned}$ & $\begin{array}{l}.050 \\
.017\end{array}$ & $\begin{array}{l}.0275 \\
.008\end{array}$ & $\begin{array}{r}-.002 \\
.008\end{array}$ \\
\hline Average.. & & & Q & $\ldots$ & -.0022 & …...... & .0205 & .0182 \\
\hline
\end{tabular}

$11 / 3$ INCHES, 16 GAUGE $\frac{L}{r}=70$.

\begin{tabular}{|c|c|c|c|c|c|c|c|c|}
\hline 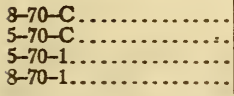 & $\begin{array}{l}0.0635 \\
.065 \\
.060 \\
.0623\end{array}$ & $\begin{array}{l}0.0615 \\
.0605 \\
.065 \\
.0630\end{array}$ & $\begin{array}{l}-0.001 \\
-.0022 \\
+.0025 \\
+.0003\end{array}$ & $\begin{array}{l}-0.0052 \\
-.011 \\
+.0131 \\
+.0015\end{array}$ & $\begin{array}{l}-0.0062 \\
-.013 \\
+.0156 \\
+.0018\end{array}$ & $\begin{array}{l}0.028 \\
.054 \\
.045 \\
.052\end{array}$ & $\begin{array}{l}0.015 \\
.0295 \\
.020 \\
.0255\end{array}$ & $\begin{array}{l}0.009 \\
.017 \\
.0356 \\
.027\end{array}$ \\
\hline $\begin{array}{l}5-70-5 \ldots \ldots \ldots \ldots \\
5-70-5 \ldots \ldots \ldots \ldots \\
8-70-20 \ldots \ldots \ldots \ldots \\
7-70-20 \ldots \ldots \ldots \ldots\end{array}$ & $\begin{array}{l}.066 \\
.064 \\
.063 \\
.059\end{array}$ & $\begin{array}{l}.050 \\
.060 \\
.063 \\
.065\end{array}$ & $\begin{array}{r}-.003 \\
-.002 \\
.000 \\
+.003\end{array}$ & $\begin{array}{l}-.0156 \\
-.0106 \\
.000 \\
+.0150\end{array}$ & $\begin{array}{l}-.0186 \\
-.0126 \\
.000 \\
+.0180\end{array}$ & $\begin{array}{l}.015 \\
.022 \\
.025 \\
.023\end{array}$ & $\begin{array}{l}.0105 \\
.013 \\
.0125 \\
.0085\end{array}$ & $\begin{array}{r}-.008 \\
.0004 \\
.0125 \\
.026\end{array}$ \\
\hline Average.. & & & & & -.0019 & & $.01 \cdot 68$ & .0149 \\
\hline
\end{tabular}

11/3 INCHES, 16 GAUGE $\frac{L}{r}=110$.

\begin{tabular}{|c|c|c|c|c|c|c|c|c|}
\hline 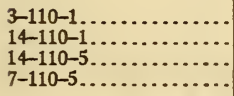 & $\begin{array}{l}0.0655 \\
.0615 \\
.062 \\
.063\end{array}$ & $\begin{array}{l}0.0610 \\
.0685 \\
.066 \\
.060\end{array}$ & $\begin{array}{l}-0.0023 \\
+.0036 \\
+.002 \\
-.0015\end{array}$ & $\begin{array}{r}-0.0121 \\
+.0182 \\
+.0105 \\
-.008\end{array}$ & $\begin{array}{l}-0.0145 \\
+.022 \\
+.0125 \\
-.0095\end{array}$ & $\begin{array}{l}0.088 \\
.040 \\
.043 \\
.048\end{array}$ & $\begin{array}{l}0.046 \\
.0166 \\
.0195 \\
.0255\end{array}$ & $\begin{array}{l}0.031 \\
.039 \\
.036 \\
.016\end{array}$ \\
\hline Average. . & & & 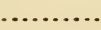 & & +.0026 & & .0269 & .0305 \\
\hline
\end{tabular}

$1 k=\frac{t_{\max }-t_{\min }}{2}$

$y=\frac{A_{1}}{A_{0}-A_{1}} k$.

${ }^{3} e_{x}=\frac{R-2 k}{2}$. 
(d) ACCURACY OF METḤOD OF DETERMINING ECCENTRICITY.

From the elastic theory one may express the relation between the lateral deflection at the middle of a column $y_{\mathrm{m}}$ and the initial eccentricity $e$ for round end columns, as follows:

$$
y_{\mathrm{m}}=e\left(\sec \sqrt{\frac{P}{E I}} \frac{L}{2}-\mathrm{I}\right)
$$

It can be shown that when the load $P$ is $4 / 9 P_{E}$-that is, $4 / 9$ of Euler's maximum load - the deflection at the center of the column is equal to the initial eccentricity, provided $P$ does not stress the material beyond the proportional limit. In Table 8 are given the eccentricities and the deflection at a load of $4 / 9$ Euler's load for the eight columns tested. There is practically exact agreement in all except column $9-\mathrm{rro}-\mathrm{C}$, indicating that the method of determining the eccentricities was very accurate.

TABLE 8.-Eccentricities of Columns.

$11 / 2$ INCHES, 20 GAUGE $\frac{L}{r}=110$.

\begin{tabular}{|c|c|c|c|c|c|c|c|c|c|}
\hline \multirow{2}{*}{ Strut number. } & \multicolumn{2}{|c|}{$\begin{array}{l}\text { Average wall } \\
\text { thickness. }\end{array}$} & \multirow{2}{*}{$k$} & \multirow{2}{*}{$y$} & \multirow{2}{*}{$\begin{array}{l}\text { Eccen- } \\
\text { tricity } \\
\text { from } \\
\text { wall } \\
\text { varia- } \\
\text { tion. } \\
e_{w}=k+y\end{array}$} & \multirow{2}{*}{$\begin{array}{c}\text { Dial de- } \\
\text { flection. } \\
R\end{array}$} & \multirow{2}{*}{$\begin{array}{l}\text { Eccen- } \\
\text { tricity } \\
\text { due to } \\
\text { crooked- } \\
\text { ness. } \\
\text { ex }\end{array}$} & \multirow{2}{*}{$\begin{array}{c}\text { Total } \\
\text { eccen- } \\
\text { tricity. } \\
e=e_{w}+e_{x}\end{array}$} & \multirow{2}{*}{$\begin{array}{l}\text { Deflec- } \\
\text { tion of } \\
\text { column } \\
\text { at load. } \\
P=\frac{4}{9} P_{\text {II }}\end{array}$} \\
\hline & $\begin{array}{l}\text { Top } \\
\text { side. }\end{array}$ & $\begin{array}{l}\text { Bottom } \\
\text { side. }\end{array}$ & & & & & & & \\
\hline 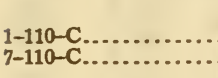 & $\begin{array}{r}\text { Inch. } \\
0.039 \\
.035\end{array}$ & $\begin{array}{r}\text { Inch. } \\
0.036 \\
.035\end{array}$ & $\begin{array}{c}\text { Inch. } \\
-0.0015 \\
.00\end{array}$ & $\begin{array}{c}\text { Inch. } \\
-0.0144 \\
.00\end{array}$ & $\begin{array}{c}\text { Inch. } \\
-0.0159 \\
.00\end{array}$ & $\begin{array}{r}\text { Inch. } \\
0.104 \\
.016\end{array}$ & $\begin{array}{l}\text { Inch. } \\
0.0535 \\
.008\end{array}$ & $\begin{array}{r}\text { Inch. } \\
0.038 \\
.008\end{array}$ & $\begin{array}{r}\text { Inch. } \\
0.040 \\
.009\end{array}$ \\
\hline
\end{tabular}

$11 / 2$ INCHES, 16 GAUGE $\frac{L}{r}=110$.

\begin{tabular}{|c|c|c|c|c|c|c|c|c|c|}
\hline $\begin{array}{l}10-110-C \\
9-110-C . . .\end{array}$ & $\begin{array}{l}0.0637 \\
.060\end{array}$ & $\begin{array}{r}0.064 \\
.064\end{array}$ & $\begin{array}{r}+0.0004 \\
+.0020\end{array}$ & $\begin{array}{r}+0.002 \\
+.010\end{array}$ & $\begin{array}{c}+0.0024 \\
+.012\end{array}$ & $\begin{array}{r}0.037 \\
.100\end{array}$ & $\begin{array}{r}0.018 \\
.048\end{array}$ & $\begin{array}{r}0.020 \\
.060\end{array}$ & $\begin{array}{r}0.019 \\
.051\end{array}$ \\
\hline
\end{tabular}

11/2 INCHES, 20 GAUGE $\frac{L}{r}=70$.

\begin{tabular}{|c|c|c|c|c|c|c|c|c|c|}
\hline 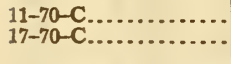 & $\begin{array}{l}0.035 \\
.0365\end{array}$ & $\begin{array}{r}0.0335 \\
.0355\end{array}$ & $\begin{array}{r}-0.0007 \\
-.0006\end{array}$ & $\begin{array}{l}-0.007 \\
-.006\end{array}$ & $\begin{array}{r}-0.0077 \\
-.0066\end{array}$ & $\begin{array}{r}0.025 \\
.029\end{array}$ & $\begin{array}{l}0.0132 \\
.015\end{array}$ & $\begin{array}{l}0.0055 \\
.008\end{array}$ & $\begin{array}{r}0.004 \\
.006\end{array}$ \\
\hline
\end{tabular}

$11 / 2$ INCHES, 16 GAUGE $\frac{L}{r}=70$.

\begin{tabular}{|c|c|c|c|c|c|c|c|c|c|}
\hline 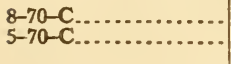 & $\begin{array}{l}0.0635 \\
.065\end{array}$ & $\begin{array}{r}0.0615 \\
.0605\end{array}$ & 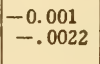 & $\begin{array}{l}-0.0052 \\
-.011\end{array}$ & -0.0062 & $\begin{array}{r}0.028 \\
.054\end{array}$ & $\begin{array}{l}0.015 \\
.0295\end{array}$ & $\begin{array}{r}0.009 \\
.017\end{array}$ & $\begin{array}{r}0.003 \\
.017\end{array}$ \\
\hline
\end{tabular}




\section{APPLICATION OF MODIFIED RATIONAL FORMULA.}

The results obtained by applying the eccentricities caused by tube irregularities to the modified rational formula

$$
f_{\mathrm{c}}=\frac{P}{A}+\frac{P e c}{I} \sec \frac{\pi}{2} \sqrt{\frac{P}{P_{\mathrm{E}}}}+\frac{w E c}{P}\left(\sec \frac{\pi}{2} \sqrt{\frac{P}{P_{\mathrm{E}}}}-\mathrm{I}\right)
$$

are given in Table 9. The maximum compressive stress $f_{\mathrm{c}}$ to cause failure of the struts subjected to combined column and transverse loading are in very close agreement with the yield point of the material. The small discrepancies that exist are on the side of safety. In the next to last column in the table are given the ultimate compressive stress $S_{\mathrm{c}}$, approximately the yield point in compression for the material, determined from the compression test of short specimen (see Table 2). The maximum compressive stress at failure $f_{\mathrm{c}}$ for the struts are in extremely close agreement with these values. The general average of the maximum compressive stress at failure for the struts is $70,600 \mathrm{lbs} . /$ in. $^{2}$ and the average ultimate compressive stress for short column, or approximate yield point in compression for the material, is 69,700 , an error of about I per cent, which is remarkably close for experimental data involving so many variables.

TABLE 9.-Results Obtained by Use of Modified Rational Formula for Combined Loading.

$11 / 2$ INCHES, 20 GAUGE $\frac{L}{r}=70$.

\begin{tabular}{|c|c|c|c|c|c|c|c|c|}
\hline Strut number. & $\begin{array}{l}\text { Trans- } \\
\text { verse } \\
\text { load. }\end{array}$ & $\begin{array}{c}\text { Maxi- } \\
\text { mum } \\
\text { bending } \\
\text { stress. } \\
S_{B}\end{array}$ & $\begin{array}{c}\text { Com- } \\
\text { pressive } \\
\text { stress } \\
\frac{P}{A}\end{array}$ & $\begin{array}{c}\text { Maxi- } \\
\text { mum } \\
\text { compres- } \\
\text { sive } \\
\text { stress at } \\
\text { failure.2 } \\
f_{0}\end{array}$ & $\begin{array}{c}\text { Yield } \\
\text { point of } \\
\text { material } \\
\text { (tension). }\end{array}$ & $\begin{array}{c}\text { Ratio. } \\
\frac{f_{0}}{Y P}\end{array}$ & $\begin{array}{c}\text { Ultimate } \\
\text { compres- } \\
\text { sive } \\
\text { stress } \\
\text { (short } \\
\text { column). } \\
S_{0}\end{array}$ & $\begin{array}{c}\text { Ratio. } \\
\frac{f_{0}}{S_{0}}\end{array}$ \\
\hline 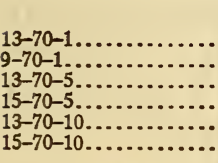 & $\begin{array}{r}\text { Pounds. } \\
1.25 \\
1.25 \\
5.0 \\
5.0 \\
10.0 \\
10.0\end{array}$ & $\begin{array}{r}\text { Lbs. } / \text { in. }^{2} \\
26,550 \\
26,800 \\
37,700 \\
38,000 \\
50,700 \\
49,700\end{array}$ & $\begin{array}{l}\text { Lbs./in. }{ }^{3} \\
41,200 \\
44,400 \\
30,100 \\
30,900 \\
22,900 \\
22,500\end{array}$ & $\begin{array}{c}\text { Lbs./in.2 }^{2} \\
67,750 \\
71,200 \\
67,800 \\
68,900 \\
73,600 \\
72,200\end{array}$ & \begin{tabular}{|c} 
Lbs./in. $^{2}$ \\
68,900 \\
66,800 \\
68,900 \\
69,900 \\
68,900 \\
69,900
\end{tabular} & $\begin{array}{r}\text { Per cent. } \\
98 \\
106 \\
98 \\
99 \\
107 \\
103\end{array}$ & $\begin{array}{c}\text { Lbs./in. }{ }^{2} \\
69,300 \\
66,400 \\
69,300 \\
68,800 \\
69,300 \\
68,800\end{array}$ & $\begin{array}{r}\text { Per cent. } \\
98 \\
107 \\
98 \\
100 \\
106 \\
105\end{array}$ \\
\hline Average.. & & & & 70,200 & 69,000 & 102 & 68,600 & 102 \\
\hline
\end{tabular}

$11 / 2$ INCHES, 20 GAUGE $\frac{L}{r}=110$.

\begin{tabular}{|c|c|c|c|c|c|c|c|c|}
\hline 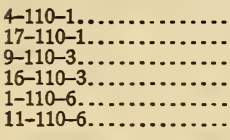 & $\begin{array}{l}1.0 \\
1.0 \\
3.0 \\
3.0 \\
6.05 \\
6.05\end{array}$ & $\begin{array}{l}50,100 \\
49,600 \\
57,500 \\
52,560 \\
61,090 \\
63,600\end{array}$ & $\begin{array}{r}19,400 \\
19,900 \\
14,300 \\
13,550 \\
8,200 \\
10,300\end{array}$ & $\begin{array}{l}69,500 \\
69,500 \\
71,800 \\
66,110 \\
69,300 \\
73,900\end{array}$ & $\begin{array}{l}63,400 \\
67,400 \\
66,800 \\
62,200 \\
61,200 \\
69,000\end{array}$ & $\begin{array}{l}109 \\
103 \\
107 \\
106 \\
113 \\
107\end{array}$ & $\begin{array}{l}65,200 \\
68,400 \\
66,400 \\
66,200 \\
64,700 \\
67,500\end{array}$ & $\begin{array}{l}106 \\
101 \\
108 \\
100 \\
107 \\
109\end{array}$ \\
\hline Average..... & ........ & & & 70,000 & 65,000 & 107 & 66,400 & 105 \\
\hline
\end{tabular}

See footnotes on p. 270 . 
TABLE 9.-Results Obtained by Use of Modified Rational Formula for Combined Loading-Continued.

$11 / 2$ INCHES, 16 GAUGE $\frac{L}{r}=70$.

\begin{tabular}{|c|c|c|c|c|c|c|c|c|}
\hline Strut number. & $\begin{array}{c}\text { Trans- } \\
\text { verse } \\
\text { load. }\end{array}$ & $\begin{array}{c}\text { Maxi- } \\
\text { mumm } \\
\text { bending } \\
\text { stress. } \\
S_{\mathrm{B}}\end{array}$ & $\begin{array}{c}\begin{array}{c}\text { Com- } \\
\text { pressive } \\
\text { stress. } \\
\frac{P}{A}\end{array} \\
\bar{A}\end{array}$ & $\begin{array}{c}\text { Maxi- } \\
\text { mum } \\
\text { compres- } \\
\text { sive } \\
\text { stress at } \\
\text { failure.2 } \\
f_{\mathrm{c}}\end{array}$ & $\begin{array}{c}\text { Yield } \\
\text { point of } \\
\text { material } \\
\text { (tension). }\end{array}$ & $\begin{array}{c}\text { Ratio. } \\
\frac{f_{0}}{Y P}\end{array}$ & $\begin{array}{l}\text { Ultimate } \\
\text { compres- } \\
\text { sive } \\
\text { stress } \\
\text { (short } \\
\text { column). } \\
S_{0}\end{array}$ & $\begin{array}{c}\text { Ratio. } \\
\frac{f_{0}}{S_{0}}\end{array}$ \\
\hline $\begin{array}{l}5-70-1 \ldots \ldots \ldots \\
8-70-1 \ldots \ldots \\
5-70-5 \ldots \ldots \\
7-70-5 \ldots \ldots \ldots \\
8-70-20 \ldots \ldots \\
7-70-20 \ldots \ldots\end{array}$ & $\begin{array}{r}\text { Pounds. } \\
1.0 \\
1.0 \\
5.0 \\
5.0 \\
20.0 \\
20.0\end{array}$ & $\begin{array}{l}\text { Lbs./in. }{ }^{2} \\
27,040 \\
27,700 \\
25,960 \\
25,780 \\
56,000 \\
54,240\end{array}$ & $\begin{array}{r}\text { Lbs./in. }{ }^{2} \\
42,900 \\
45,500 \\
42,500 \\
39,300 \\
23,250 \\
19,780\end{array}$ & \begin{tabular}{|r|} 
Lbs./in. ${ }^{2}$ \\
69,940 \\
73,200 \\
68,460 \\
65,080 \\
79,250 \\
74,000
\end{tabular} & $\begin{array}{r}\text { Lbs./in. } .^{2} \\
70,000 \\
70,200 \\
70,000 \\
67,500 \\
70,200 \\
67,500\end{array}$ & \begin{tabular}{|r|} 
Per cent. \\
100 \\
104 \\
98 \\
96 \\
113 \\
109
\end{tabular} & \begin{tabular}{|r} 
Lbs./in. ${ }^{2}$ \\
71,600 \\
74,800 \\
71,600 \\
69,300 \\
74,800 \\
69,300
\end{tabular} & $\begin{array}{r}\text { Per cent. } \\
98 \\
98 \\
96 \\
94 \\
106 \\
107\end{array}$ \\
\hline Average. & & & & 71,650 & 69,200 & 103 & 71,900 & 100 \\
\hline
\end{tabular}

$11 / 2$ INCHES, 16 GAUGE $\frac{L}{r}=110$.

\begin{tabular}{|c|c|c|c|c|c|c|c|c|}
\hline $\begin{array}{l}3-110-1 \ldots \ldots \ldots . \\
14-110-1 \ldots \ldots \ldots \\
14-110-5 \ldots \ldots \ldots \\
7-110-5 \ldots \ldots \ldots . .\end{array}$ & $\begin{array}{l}1.0 \\
1.0 \\
5.0 \\
5.0\end{array}$ & $\begin{array}{l}60,200 \\
44,770 \\
54,740 \\
53,810\end{array}$ & $\begin{array}{l}21,100 \\
19,870 \\
14,050 \\
14,250\end{array}$ & $\begin{array}{l}81,300 \\
64,700 \\
68,800 \\
68,060\end{array}$ & $\begin{array}{l}71,300 \\
64,600 \\
64,600 \\
67,500\end{array}$ & $\begin{array}{l}114 \\
100 \\
106 \\
100\end{array}$ & $\begin{array}{l}80,400 \\
68,800 \\
68,800 \\
69,300\end{array}$ & $\begin{array}{r}101 \\
94 \\
100 \\
98\end{array}$ \\
\hline Average.. & $\ldots$ & & & 70,700 & 67,000 & 105 & 71,800 & 98 \\
\hline
\end{tabular}

${ }^{1} S_{\mathrm{B}}=\frac{M_{\mathrm{O}}}{\frac{I}{c}}=\frac{P e c}{I} \sec \frac{\pi}{2} \sqrt{\frac{P}{P_{\mathrm{B}}}}+\frac{w E c}{P}\left(\sec \frac{\pi}{2} \sqrt{\frac{P}{P_{\mathrm{B}}}}-\mathrm{x}\right)$.

${ }^{2} f_{\mathrm{c}}=\frac{M_{\mathrm{o}}}{\frac{I}{C}}+\frac{P}{A}$

The accuracy and safety with which this modified rational formula determines the stress and strength of struts subjected to transverse loading is graphically shown in the right half of Figure Io. In this figure the same values for abscissas are used as in the left half of the figure. The ordinate values $f_{c}$, the maximum compressive stresses at failure, were computed by the modified formula. It will be seen that the small variations which exist are less than the variations in the short compression tests. The two high values are for tubing Nos. 3 and 8 , which had a high yield point in compression.

The relation between the maximum compressive stress at failure determined by the formula and the yield point of the material is shown in Figure 15 . The numbers assigned to the different sections of tubing used in these tests are plotted as abscissas and the maximum compressive stress at failure for the struts cut from these sections as ordinates. The heavy line connects the value of ultimate or maximum compressive stress, approximate yield point in compression obtained from short 
compression tests of each section of tubing. This figure also shows the variation in per cent of maximum compressive stress from the yield point of the material. It will be noted that the failing stress of struts computed by the formula agrees very closely with the failing stress of the short compression pieces.

Theoretically, the modified formula is not exact above the proportional limit, as the formula involves the modulus of elasticity $E$. In this investigation the material used had very nearly the same proportional limit and yield point values. The assumption of strict proportionality between stress and strain up to failure, although not theoretically exact, gives results that agree within a very small percentage error with actual conditions, and for all practical purposes can be used in design.

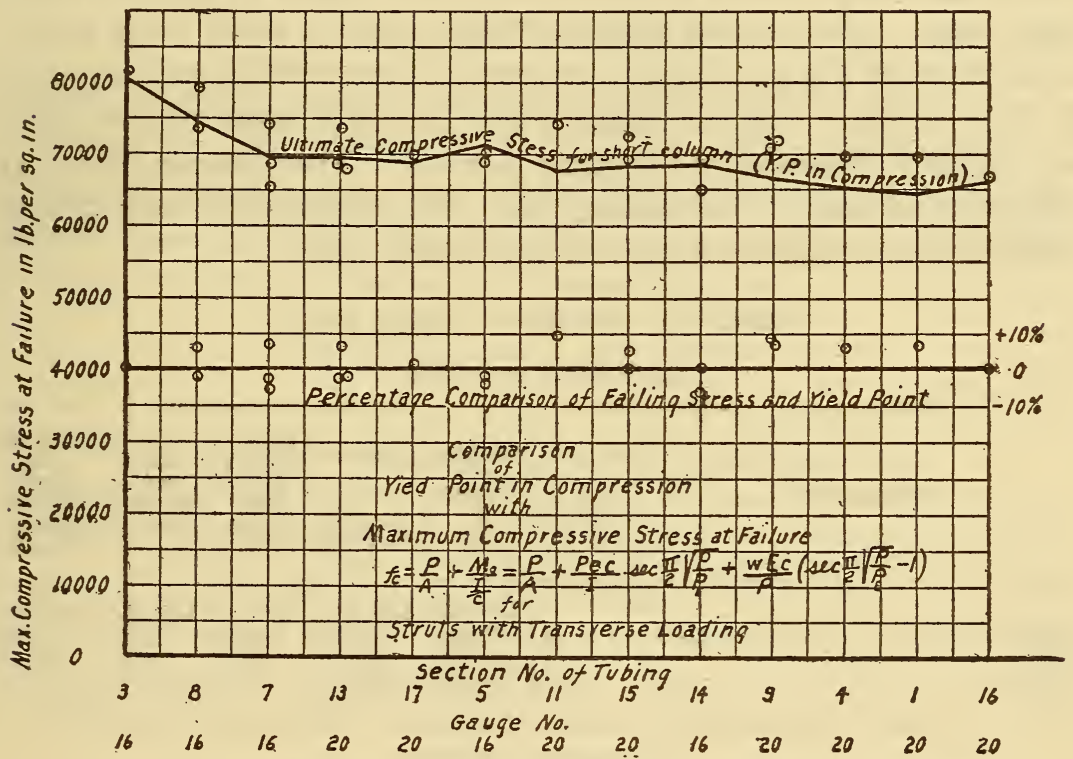

FIG. I5.-Comparison of the maximum compressive stress at failure by modified rational formula with the yield point of the material.

\section{APPLICATION OF "SECANT" COLUMN FORMULA.}

(a) TO COLUMNS.

The modified rational formula may be readily applied to columns to determine the maximum compressive stress to cause failure. By this formula the maximum intensity of compressive stress is

$$
f_{\mathrm{o}}=\frac{P}{A}+\frac{P e c}{I} \sec \frac{\pi}{2} \sqrt{\frac{P}{P_{\mathrm{E}}}}+\frac{w E c}{P}\left(\sec \frac{\pi}{2} \sqrt{\frac{P}{P_{\mathrm{E}}}}-\mathrm{I}\right)
$$


For columns the transverse load $w$ is zero, so that the above formula becomes

$$
f_{\mathrm{c}}=\frac{P}{A}+\frac{P e c}{I} \sec \frac{\pi}{2} \sqrt{\frac{\bar{P}}{P_{\mathrm{E}}}}
$$

or

$$
f_{\mathrm{c}}=\frac{P}{A}\left(\mathrm{I}+\frac{e c}{r^{2}} \sec \frac{L}{2} \sqrt{\frac{P}{E I}}\right)
$$

which is the well-known "secant" formula for columans with eccentric loading.

The results of the column tests are given in Table ro. The eccentricities about the horizontal axis have been used to determine the maximum compressive stress for the shorter columns. The long columns with an $L / r$ ratio of I Io failed at Euler's maximum load. The results indicate that there is fairly close agreement between the maximum compressive stress at failure computed by the "secant" column formula and the yield point of the material with the exception of column $5-70-C$. This column failed by deflecting sideways, indicating that the eccentricity was greater than the value taken about the horizontal axis.

TABLE 10.-Results of Column Tests.

\begin{tabular}{|c|c|c|c|c|c|c|}
\hline Strut number. & $\underset{r a t i o .}{\frac{L}{r}}$ & $\begin{array}{l}\text { Column } \\
\text { unit } \\
\text { stress. } \\
\frac{P}{A}\end{array}$ & $\begin{array}{c}\text { Euler } \\
\frac{P}{A}=\frac{\pi^{2} E}{\left(\frac{L}{r}\right)^{2}}\end{array}$ & $\begin{array}{l}\text { Eccen- } \\
\text { tricity e } \\
\text { about } \\
\text { hori- } \\
\text { zontal } \\
\text { axis. }\end{array}$ & $\begin{array}{c}\text { Maxi- } \\
\text { mum } \\
\text { com- } \\
\text { pressive } \\
\text { stress.1 } \\
f_{0}\end{array}$ & $\begin{array}{l}\text { Ultimate } \\
\text { com- } \\
\text { pressive } \\
\text { stress } S_{\text {. }} \\
\text { (short } \\
\text { column). }\end{array}$ \\
\hline $\begin{array}{r}11-70-C \\
17-70-C \\
1-110-C \\
7-110-C \\
11\end{array}$ & $\begin{array}{r}70 \\
70 \\
110 \\
110\end{array}$ & $\begin{array}{r}\text { Lbs./in. }{ }^{3} \\
52,900 \\
51,930 \\
23,200 \\
24,000\end{array}$ & $\begin{array}{r}\text { Lbs./in. }{ }^{2} \\
58,500 \\
58,400 \\
23,600 \\
23,700\end{array}$ & $\begin{array}{l}\text { Inch. } \\
0.0055 \\
.008 \\
0 .\end{array}$ & $\begin{array}{r}\text { Lbs./in. }{ }^{2} \\
71,400 \\
72,400\end{array}$ & $\begin{array}{r}\text { Lbs./in.2 } \\
67,600 \\
68,400 \\
\ldots\end{array}$ \\
\hline
\end{tabular}

$1 \frac{1}{2}$ INCHES, 20 GAUGE.

\begin{tabular}{|c|c|c|c|c|c|c|}
\hline $\begin{array}{l}8-70-C \\
5-70-C \\
10-110-C \\
9-110-C .\end{array}$ & $\begin{array}{r}70 \\
70 \\
110 \\
110\end{array}$ & $\begin{array}{l}52,800 \\
47,650 \\
23,500 \\
22,500\end{array}$ & $\begin{array}{l}58,100 \\
58,300 \\
23,500 \\
23,500\end{array}$ & $\begin{array}{r}0.009 \\
.0017 \\
\cdots \\
\cdots\end{array}$ & $\begin{array}{r}72,300 \\
63,600 \\
\cdots\end{array}$ & $\begin{array}{r}74,800 \\
71,600 \\
\cdots \\
\cdots\end{array}$ \\
\hline
\end{tabular}

11/2 INCHES, 16 GAUGE.

$$
{ }_{1}^{1} f_{0}=\frac{P}{A}\left(\mathrm{r}+\frac{e c}{r^{2}} \sec \frac{\pi}{2} \sqrt{\frac{P}{P_{\mathrm{E}}}}\right) .
$$

(b) TO STRUTS WITH TRANSVERSE LOADING.

The "secant" column formula can be applied to struts subjected to transverse loading with safety and a reasonable degree of accuracy if certain modifications in the determination of the effective eccentricity are made. Consider, first, a strut to be 
under a very small end load and subjected to a uniform transverse load of $w$ pounds per linear inch. The strut, by the deflection formula for a uniform transverse load, will be deflected at the center a distance

$$
e_{\mathrm{B}}=\frac{5}{384} \frac{w L^{4}}{E I}
$$

and the bending (flexual) stress $S_{\mathrm{B}}$ in the extreme fiber at the mid-length section resulting from the transverse loading will be

$$
S_{\mathrm{B}}=\frac{\mathrm{I}}{8} \frac{w L^{2} c}{I}
$$

On application of the end load the section at the mid length of the strut will have an effective eccentricity $e$ with reference to the line of load of

$$
e=e_{\mathrm{o}}+e_{\mathrm{B}}=e_{\mathrm{o}}+\frac{5}{384}-\frac{w L^{4}}{E I}
$$

where $e_{0}$ is the original eccentricity of the strut in the plane of deflection due to irregularities in the tube, and $e_{B}$ is the deflection of the tube produced by the transverse loading. The maximum column stress $S_{u}$ in the extreme fiber of the section at the middle of the strut is by the "secant" column formula

$$
S_{\mathrm{u}}=\frac{P}{A}\left(\mathrm{I}+\frac{e c}{r^{2}} \sec \frac{\pi}{2} \sqrt{\frac{\bar{P}}{P_{E}}}\right)
$$

where $e$ is the effective eccentricity given in equation (3).

The maximum compressive stress at the extreme fiber of the mid section is the sum of the maximum column stress $S_{u}$ and the bending (flexual) stress $S_{\mathrm{B}}$. It is to be expected that the strut will fail when the sum of these stresses is approximately equal to the yield point of the material.

The maximum compressive stress at failure is therefore

$$
f_{\mathrm{c}}=S_{\mathrm{u}}+S_{\mathrm{B}}=\text { yield point (approximately) }
$$

or

$$
f_{\mathrm{c}}=\frac{P}{A}\left(\mathrm{I}+\frac{e c}{r^{2}} \sec \frac{\pi}{2} \sqrt{\frac{P}{P_{\mathrm{E}}}}\right)+\frac{\mathrm{I}}{8} \frac{w L^{2} c}{I}
$$

the effective eccentricity $e$ to be taken as the sum of the original eccentricity and the deflection of the strut resulting from the transverse load.

The results obtained by applying the above formula to the combined tests are given in Table I I. The table shows that this method of computation gives values that are, on the average, about 6 per cent higher than those determined by the more exact 
formula. The error, however, is on the side of safety. Where extreme accuracy is not required, this formula can be safely used and is more reliable for designing than Perry's formula, which neglects the effect of eccentricities.

TABLE 11.-Results Obtained by Applying "Secant" Column Formula for Combined Loading.

$11 / 2$ INCHES, 20 GAUGE $\frac{L}{r}=70$.

\begin{tabular}{|c|c|c|c|c|c|c|c|}
\hline Strut number. & $\begin{array}{c}\text { Trans- } \\
\text { verse } \\
\text { load. }\end{array}$ & $\begin{array}{c}\text { Effective } \\
\text { eccen- } \\
\text { tricity. } \\
e_{0}+e_{B} i\end{array}$ & $\begin{array}{c}\text { Maxi- } \\
\text { mum } \\
\text { column } \\
\text { stress. } \\
S_{\mathrm{u}^{2}}\end{array}$ & $\begin{array}{l}\text { Bending } \\
\text { stress } \\
\text { due to } \\
\text { trans- } \\
\text { verse } \\
\text { load. } \\
S_{B_{B}}\end{array}$ & $\begin{array}{l}\text { Maxi- } \\
\text { mum } \\
\text { compres- } \\
\text { sive } \\
\text { stress at } \\
\text { failure. } \\
f_{0}^{4}\end{array}$ & $\begin{array}{l}\text { Ultimate } \\
\text { compres- } \\
\text { sive } \\
\text { stress } \\
\text { (short } \\
\text { column). } \\
S_{0}\end{array}$ & $\begin{array}{c}\text { Ratio. } \\
\frac{f_{0}}{S_{0}}\end{array}$ \\
\hline 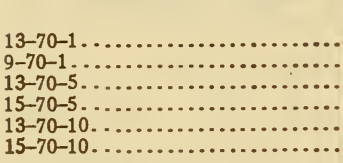 & $\begin{array}{r}\text { Pounds. } \\
1.25 \\
1.25 \\
5.0 \\
5.0 \\
10.0 \\
10.0\end{array}$ & $\begin{array}{l}\text { Inch. } \\
0.058 \\
.0405 \\
.126 \\
.121 \\
.198 \\
.199\end{array}$ & $\begin{array}{r}\text { Lbs./in. }{ }^{2} \\
67,700 \\
69,300 \\
55,400 \\
56,200 \\
46,100 \\
44,700\end{array}$ & $\begin{array}{r}\text { Lbs. } \text { in. }^{2} \\
3,600 \\
3,600 \\
14,600 \\
14,900 \\
29,400 \\
29,200\end{array}$ & $\begin{array}{r}\text { Lbs. } / \text { in. }^{2} \\
71,300 \\
72,900 \\
70,000 \\
71,100 \\
75,500 \\
73,900\end{array}$ & $\begin{array}{c}\text { Lbs. } / \text { in. }^{2} \\
69,300 \\
66,400 \\
69,300 \\
68,800 \\
69,300 \\
68,800\end{array}$ & $\begin{array}{r}\text { Per cent. } \\
103 \\
110 \\
101 \\
103 \\
109 \\
107\end{array}$ \\
\hline Average. . & & & & & 72,400 & 68,600 & 105 \\
\hline
\end{tabular}

$11 / 2$ INCHES, 20 GAUGE $\frac{L}{r}=110$.

\begin{tabular}{|c|c|c|c|c|c|c|c|}
\hline 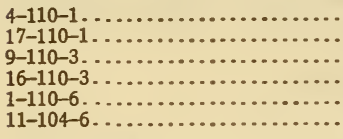 & $\begin{array}{l}1.0 \\
1.0 \\
3.0 \\
3.0 \\
6.05 \\
6.05\end{array}$ & $\begin{array}{l}0.135 \\
.119 \\
.358 \\
.347 \\
.644 \\
.542\end{array}$ & $\begin{array}{l}69,400 \\
70,800 \\
56,200 \\
49,500 \\
31,900 \\
38,200\end{array}$ & $\begin{array}{r}7,000 \\
6,900 \\
20,700 \\
20,800 \\
39,500 \\
38,000\end{array}$ & $\begin{array}{l}76,400 \\
77,700 \\
76,900 \\
70,300 \\
71,400 \\
76,200\end{array}$ & $\begin{array}{l}65,200 \\
68,400 \\
66,400 \\
66,200 \\
64,700 \\
67,600\end{array}$ & $\begin{array}{l}117 \\
113 \\
116 \\
106 \\
110 \\
113\end{array}$ \\
\hline Average. & & & & & 74,800 & 66,400 & 112 \\
\hline
\end{tabular}

$11 / 2$ INCHES, 16 GAUGE $\frac{L}{r}=70$.

\begin{tabular}{|c|c|c|c|c|c|c|c|}
\hline 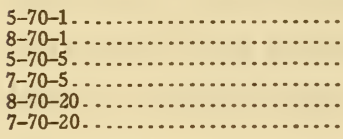 & $\begin{array}{r}1.0 \\
1.0 \\
5.0 \\
5.0 \\
20.0 \\
20.0\end{array}$ & $\begin{array}{r}0.045 \\
.037 \\
.042 \\
.050 \\
.212 \\
.225\end{array}$ & $\begin{array}{l}68,900 \\
73,800 \\
65,000 \\
59,650 \\
49,400 \\
41,400\end{array}$ & $\begin{array}{r}1,660 \\
1,630 \\
7,900 \\
8,130 \\
32,500 \\
33,700\end{array}$ & $\begin{array}{l}70,560 \\
75,400 \\
72,900 \\
67,800 \\
81,900 \\
75,100\end{array}$ & $\begin{array}{l}71,600 \\
74,800 \\
71,600 \\
69,300 \\
74,800 \\
69,300\end{array}$ & $\begin{array}{r}98 \\
101 \\
102 \\
98 \\
109 \\
108\end{array}$ \\
\hline Average................ & & & & & 72,300 & 71,900 & 102 \\
\hline
\end{tabular}

$11 / 2$ INCHES, 16 GAUGE $\frac{L}{r}=110$.

\begin{tabular}{|c|c|c|c|c|c|c|c|}
\hline 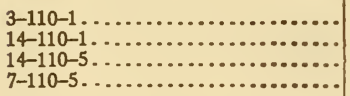 & $\begin{array}{l}1.0 \\
1.0 \\
5.0 \\
5.0\end{array}$ & $\begin{array}{r}0.091 \\
.099 \\
.337 \\
.327\end{array}$ & $\begin{array}{l}85,700 \\
66,200 \\
54,100 \\
53,300\end{array}$ & $\begin{array}{r}3,900 \\
4,000 \\
20,100 \\
20,300\end{array}$ & $\begin{array}{l}89,600 \\
70,200 \\
74,100 \\
73,600\end{array}$ & $\begin{array}{l}80,400 \\
68,800 \\
68,800 \\
69,300\end{array}$ & $\begin{array}{l}111 \\
102 \\
107 \\
106\end{array}$ \\
\hline Average................ & & & & 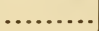 & 76,800 & 71,800 & 106 \\
\hline
\end{tabular}

\footnotetext{
$e \mathrm{~B}=\frac{5}{384} \frac{W L^{4}}{E} \frac{1}{}$

${ }^{2} S_{\mathrm{u}}=\frac{P}{A}\left(\mathrm{I}+\frac{e c}{r^{2}} \sec \frac{\pi}{2} \sqrt{\frac{P}{P_{\mathrm{D}}}}\right)$.

${ }^{3} S_{\mathrm{B}}=\frac{I}{8} \frac{w L^{2} c}{I}$.

${ }^{4} f_{\mathrm{c}}=S_{\mathrm{u}}+S_{\mathrm{B}}$.
}

${ }^{1} e_{0}=$ original eccentricity due to tube irregularities. 


\section{CONCLUSIONS.}

The results of this investigation warrant the following conclusions:

I. For determining the strength of a strut account must be taken of the effect of eccentricity. For steel tubing struts the eccentricity resulting from (a) variation in wall thickness and (b) deviation from straightness are very important factors in determining the strength.

2. For struts subjected to combined column and transverse loading it is assumed that failure occurs when the maximum compressive fiber stress approximates the yield point of the material. The commonly used formulas which neglect the effect of eccentricity are

$$
\begin{aligned}
& f_{\mathrm{c}}=\frac{P}{A}+\frac{M_{o}}{\frac{I}{c}}=\frac{P}{A}+\frac{w E c}{P}\left(\sec \frac{\pi}{2} \sqrt{\frac{P}{P_{\mathrm{E}}}}-\mathrm{I}\right) \\
& f_{\mathrm{c}}=\frac{P}{A}+\frac{M_{o}}{\frac{I}{c}}=\frac{P}{A}+\frac{c}{I} \frac{w L^{2}}{8}\left(\frac{P_{\mathrm{E}}}{P_{\mathrm{E}}-P}\right) .
\end{aligned}
$$

These do not represent actual strut condition and are not confirmed by experimental data. The use of these formulas for design purposes is shown by the data of this investigation to be inadvisable and possibly dangerous, especially for short struts or struts with small transverse loads.

3. A modified rational formula based upon consideration of the effect of eccentricity of loading,

$$
f_{\mathrm{c}}=\frac{P}{A}+\frac{M_{o}}{\frac{I}{c}}=\frac{P}{A}+\frac{P e c}{I} \sec \frac{\pi}{2} \sqrt{\frac{P}{P_{\mathrm{E}}}}+\frac{w E c}{P}\left(\sec \frac{\pi}{2} \sqrt{\frac{P}{P_{\mathrm{E}}}}-\mathrm{I}\right)
$$

was found to fit the experimental results very closely, the agreement being such as to indicate that it is the preferable formula for design where accuracy and safety are essential.

4. Failure of a strut subjected to combined column and transverse loading will occur when the maximum compressive stress $f_{\mathrm{c}}$ computed by this formula is approximately equal to the yield point of the material.

5. The results of the few tests made on tubes as columns indicate that failure of a column will occur when the extreme fiber stress is equal to the yield point of the material. For column loading the modified rational formula also applies, as it reduces to the "secant" column formula

$$
f_{\mathrm{c}}=\frac{P}{A}\left(\mathrm{I}+\frac{e c}{r^{2}} \sec \frac{\pi}{2} \sqrt{\frac{P}{P_{\mathrm{E}}}}\right)
$$

for such conditions of loading, since the transverse load $w$ is zero. 
6. A safe and reasonably accurate computation of stress for strut under transverse loading can be obtained by summing the bending stress $S_{\mathrm{B}}$ due to the transverse load as computed by the ordinary formula $\left(S_{\mathrm{B}}=\frac{\mathrm{I}}{8} \frac{w L^{2} c}{I}\right)$ and the column stress $S_{\mathrm{u}}$ obtained by the "secant" column formula. For failure

$$
f_{\mathrm{c}}=S_{\mathrm{B}}+S_{\mathrm{u}}=\frac{\mathrm{I}}{8} \frac{w L^{2} c}{I}+\frac{P}{A}\left(\mathrm{I}+\frac{e c}{r^{2}} \sec \frac{\pi}{2} \sqrt{\frac{P}{P_{\mathrm{E}}}}\right)
$$

approximates the yield point of the material, where the effective eccentricity $e$ is the sum of the original eccentricity $e_{\mathrm{c}}$ due to tube irregularities, and the deflection at the middle of the strut $e_{\mathrm{B}}$ due to the applied transverse load, the latter for a uniformly distributed load being $e_{\mathrm{B}}=\frac{5}{384} \frac{w L^{4}}{E I}$. The results obtained by this formula are shown by the data to be on the average about 6 per cent too high; the error, however, is on the side of safety.

\section{RECOMMENDATIONS.}

I. The results show that there exists quite a wide deviation from straightness and wide variation in wall thickness in commercial tubing. Differences in wall thickness may cause variation in the area of two different pieces of tubing of the same gauge and diameter of 8 per cent, with corresponding variation in other properties. The data also show that stresses produced by eccentricities resulting from these variations are in some cases very high and unless known and considered are liable to be dangerous. These variables should, therefore, preferably be limited to as narrow a range as possible in the specifications for commercial tubing and enforced by careful and rigid inspection.

2. A new empirical formula for steel-tubing struts under transverse loading may be obtained by assigning a numerical value to the effective eccentricity in the modified rational formula. This value may be determined either by average measurements of commercial tubing or from the limits stated in the specification. With this procedure, the formula would probably be of assistance for design purposes when the actual eccentricity can not be determined.

3. The same numerical value for eccentricity, determined and corroborated by further experiments on columns, when applied to the "secant" formula, would probably give a satisfactory and accurate column formula for design purposes.

WASHINGTON, February Io, I 924. 